H. URABE AND C.C. YANG

KODAI MATH. J.

3 (1980), $253-286$

\title{
ON A CHARACTERISTIC PROPERTY OF PERIODIC ENTIRE FUNCTIONS
}

\author{
By Hironobu Urabe and Chung-Chun Yang
}

Introduction. We shall pursue an investigation on a certain functional equation treated in [8] with some overmuch restrictions. The functional equation is related to the following problem: If two entire functions in a certain class have the same zero-sets (including multiplicities), then what can be said about these functions?

Denoting by $\boldsymbol{G}(b)$ the class of all enture functions each of which is periodic with period $b(\neq 0)$ mod a non-constant entire function of order less than one (cf. Def. in $\S 1$ ), in this paper, we shall prove that if two entire functions belonging to $G\left(b_{\jmath}\right)(\jmath=1,2)$ have the same zero-sets (essentially), then they must coincide up to a non-zero multiplicative constant (Theorem 1). In the proof, we use the Borel-Nevanlinna type unicity theorem.

Note that, together with $\boldsymbol{G}(b)$, the class $\boldsymbol{J}(b)$, consisting of the entire functions each of which is periodic mod a non-constant polynomial of degree one, is significant in factorization theory (under composition) of transcendental entire functions (cf. for example, [1] or [7]).

Now recall some of the results of Gross ([2]). Among others, he proved that any non-constant, periodic, entire function $H(z)$ has an infinite number of fixed points, that is, the zeros of $H(z)-z$. Further the fixed points play an important role especially in cases concerning periodic entire functions (in factorization theory, etc.). So one might expect that periodic entire functions would be uniquely determined by the sets of their fixed points. In this paper, we'll show that this is the case (Theorem 2).

Here, the first author wishes to express his gratitude to Professors Y. Kusunoki (Kyoto Lniv.) and M. Ozawa (Tokyo Inst. of Technology) for their encouragement and suggestions.

\section{Statement of results.}

At first, we'll give the definition of the class $\boldsymbol{G}(b)$ explicitly.

Definition. For a non-zero constant $b$, we denote by $G(b)$ the class of entire functions of the form;

Received April 16, 1979 


$$
f(z)=h(z)+H(z),
$$

where $H(z)$ and $h(z)$ are non-constant entire functions such that $H(z)$ is periodic with period $b, H(z+b) \equiv H(z)$, and that $h(z)$ is of order less than one.

After we've written a short summary [9], we get a generalization. Our generalized results are stated as follows.

THEOREM 1. Let $f(z) \in \boldsymbol{G}\left(b_{1}\right)$ and $g(z) \in \boldsymbol{G}\left(b_{2}\right)$ for some non-zero constants $b_{3}$ $(\jmath=1,2)$. Assume that the sets of the zeros of $f(z)$ and $g(z)$ are identical (including multiplicities) except at most a (sequence) set whose exponent of convergence is less than one. Then we must have

$$
f(z) \equiv c \cdot g(z)
$$

for some non-zero constant $c$ and $b_{1} / b_{2}$ is a rational number.

Remark. Let $f(z) \in \boldsymbol{G}\left(b_{1}\right)$ and $g(z) \in \boldsymbol{G}\left(b_{2}\right)$ be represented as

$$
f(z)=h(z)+H(z), \quad g(z)=k(z)+K(z),
$$

where $H\left(z+b_{1}\right) \equiv H(z), \quad K\left(z+b_{2}\right) \equiv K(z), \quad h(z)$ and $k(z)$ are non-constant entire functions such that $h$ and $k$ are both of order less than one. Then the condition of Theorem 1 concerning the zero-sets of $f$ and $g$ means that the identical relation

$$
h(z)+H(z)=(k(z)+K(z)) R(z) e^{p(z)}
$$

is valid for some meromorphic function $R(z)$ (決) of order less than one and for some entire function $p(z)$.

The conclusion (1) is equivalent to show that $R(z)$ and $p(z)$ are both constant.

Also note that any meromorphic function $R(z)(\not \equiv 0)$ of order less than one can be represented as

$$
R(z)=v(z) / u(z)
$$

for some entire functions $u(z)$ and $v(z)$ ( $\equiv \equiv$ and without common zeros), both of which are of order less than one.

Periodic entire functions have the following characteristic property, as is mentioned in Introduction.

THEOREM 2. Let $H_{j}(z)(j=1,2)$ be non-constant pernodic entrre functions with period $b_{0}$ (resp.). Assume that the sets of the fixed points of $H_{3}(z)$ are identical (including multiplicities) except at most a (sequence) set whose exponent of convergence is less than one. Then we have necessarily that

$$
H_{1}(z) \equiv H_{2}(z)
$$

and $b_{1} / b_{2}$ is a rational number. 


\section{Lemmas.}

For the proof of Theorem 1, we shall need the following unicity theorem of Borel-Nevanlinna type due to Niino-Ozawa [5].

LEMMA A. Let $G_{j}(z)$ be a transcendental entire function and $c_{j}$ be a non-zero constant, and let $g(z)$ be an entire function, $\neq \equiv 0$ such that $T(r, g)=o\left(T\left(r, G_{j}\right)\right)$ as $r$ tends 10 infinity for any $\mathrm{J}$ with $1 \leqq J \leqq n$. Assume that there exists an identical relation such as

$$
\sum_{j=1}^{n} c_{j} G_{j}(z)=g(z)
$$

then we have necessarily

$$
\sum_{j=1}^{n} \delta\left(0, G_{\jmath}\right) \leqq n-1
$$

Here $T(r, *)$ and $\delta(a, *)$ denote the Nevanlinna characteristıc function and deficrency, respectively.

Remark. Compared with the original Niino-Ozawa's lemma, the above Lemma A will seem slightly general. But the proof is essentially same.

Also we use the following simple fact.

LEMMA B. Let $w(z)$ be a meromorphic function of order less than one and assume that

$$
w(z+b) \equiv e^{c} \cdot w(z)
$$

for some constants $b(\neq 0)$ and $c$. Then $w(z)$ must be constant.

For completeness, we prove this. Indeed, if we consider the function

$$
F(z)=w(z) \cdot \exp \left[-\frac{c}{b} z\right]
$$

then the assumption (6) implies

$$
F(z+b) \equiv F(z) .
$$

If $w(z)$ is non-constant, then $w(z)$ has at least one zero or one pole, since $w(z)$ is of order less than one by assumption. Then from (7) and (8), the exponent of convergence of the zeros or poles of $w(z)$ cannot be less than one, so that the order of $w(z)$ cannot be so. This is a contradiction. Hence $w(z)$ must be constant.

3. To prove Theorem 1, at the first step, we note here the following facts.

Proposition 1. Let the identrty (3) be valid. And assume that $p(z)$ is con- 
stant. Then $R(z)$ is constant $(\neq 0)$ and $b_{1} / b_{2}$ is a rational number.

Proposition 2. Let the identity (3) be valid. And assume that $b_{1} / b_{2}$ is a rational number. Then $R(z)$ and $p(z)$ are constants.

Proof of Prop. 1. In this case, the identity (3) may be written as

$$
h(z)+H(z)=(k(z)+K(z)) R(z) .
$$

We shall introduce here the notations;

$$
\begin{aligned}
& h_{\jmath}(z)=h\left(z+j b_{1}\right), \quad k_{\jmath}(z)=k\left(z+j b_{1}\right), \\
& R_{\jmath}(z)=R\left(z+j b_{1}\right),
\end{aligned}
$$

for any natural number $\jmath$.

Since $H\left(z+b_{1}\right)=H(z)$ by assumption, noting (10), from (9) we have

so that

$$
h_{1}-h=\left(k_{1}+K\left(z+b_{1}\right)\right) R_{1}-(k+K(z)) R,
$$

$$
K\left(z+b_{1}\right) R_{1}-K(z) R=\left(h_{1}-h\right)-\left(k_{1} R_{1}-k R\right) .
$$

Setting

$$
S(z)=R\left(z+b_{2}\right), \quad r(z)=h\left(z+b_{2}\right), \quad s(z)=k\left(z+b_{2}\right),
$$

and noting $K\left(z+b_{2}\right)=K(z)$, from (11) we obtain

$$
K\left(z+b_{1}\right) S_{1}-K(z) S=\left(r_{1}-r\right)-\left(s_{1} S_{1}-s S\right) .
$$

Here we put

$$
\begin{aligned}
& r_{\jmath}(z)=r\left(z+j b_{1}\right), \quad s_{\jmath}(z)=s\left(z+j b_{1}\right), \\
& S_{j}(z)=S\left(z+j b_{1}\right)
\end{aligned}
$$

for any natural number $j$ as in (10). Note that

$$
R_{1}\left(z+b_{2}\right)=R\left(z+b_{1}+b_{2}\right)=S\left(z+b_{1}\right)=S_{1}(z)
$$

etc., by (10), (12) and (14).

Cancelling $K\left(z+b_{1}\right)$ from the identities (11) and (13), we get

$$
\begin{aligned}
\left(R S_{1}-R_{1} S\right) K(z)= & R_{1}\left[\left(r_{1}-r\right)-\left(s_{1} S_{1}-s S\right)\right] \\
& -S_{1}\left[\left(h_{1}-h\right)-\left(k_{1} R_{1}-k R\right)\right] .
\end{aligned}
$$

Now the right hand side of this identity is of order less than one, while if $\left(R S_{1}-R_{1} S\right)$ is not identically zero, the left hand side is of order not less than one, since $K(z)$ is a non-constant periodic entire function (by assumption). Therefore we conclude

$$
R S_{1}-R_{1} S \equiv 0 \text {, or } S / R-S_{1} / R_{1} \equiv 0 \text {. }
$$

Noting (10) and (14), this means that $S / R$ is periodic with period $b_{1}$. Since $S / R$ is a meromorphic function of order less than one, an application of Lemma $B$ 
gives

$$
S / R=\text { const. }=c, \text { or } \quad c R-S \equiv 0 .
$$

Setting $R(z)=v(z) / u(z)$ as in (4), then (16) can be written as

$$
c u\left(z+b_{2}\right) v(z)-u(z) v\left(z+b_{2}\right) \equiv 0 \text {. }
$$

Assume that $R(z)$ is non-constant. Then we may assume without loss of generality that $u(z)$ is non-constant. In this case, since $u(z)$ is of order less than one, $u(z)$ has zeros. Assume $u\left(z_{0}\right)=0$. From (17), dividing the relation by $u(z)$, we have the identity

$$
c u\left(z+b_{2}\right) v(z) / u(z)=v\left(z+b_{2}\right) .
$$

Here the right hand side of (18) is entire, and since $u(z)$ and $v(z)$ have no common zeros, $u\left(z_{0}\right)=0$ implies $u\left(z_{0}+b_{2}\right)=0$, and so again by (18), changing the variable if necessary, $u\left(z_{0}+2 b_{2}\right)=0$. Repeating this argument, we obtain that

$$
u\left(z_{0}+m b_{2}\right)=0 \text {, for any natural number } m \text {. }
$$

Then from (19) we conclude that the exponent of convergence of the zeros of $u(z)$ is not less than one, and hence the order of $u(z)$ is so. This is a contradiction. Thus we have proved that $R(z)$ is constant.

Putting $R(z)=$ const. $=c(\neq 0)$, from $(11)$ we have (since $R_{1}(z)=$ const. $=c$ also)

$$
c\left(K\left(z+b_{1}\right)-K(z)\right)=\left(h_{1}-h\right)-c\left(k_{1}-k\right) .
$$

Here the left hand side of $(20)$ is periodic with period $b_{2}$, while the right hand side is of order less than one, so that we conclude again that

$$
K\left(z+b_{1}\right)-K(z)=\text { const. }
$$

Then $K\left(z+b_{2}\right)=K(z)$ and (21) imply that $K^{\prime}(z)$ (the first derived function of $K(z)$, non-constant since $K(z)$ is non-constant and periodic) is periodic with periods $b_{1}$ and $b_{2}$. Since non-constant entire function cannot be doubly periodic, $b_{1} / b_{2}$ must be a rational number, which is to be proved.

Proof of Prop. 2. By the assumption, we may put

$$
b=m b_{1}=n b_{2}
$$

for some non-zero integers $m$ and $n$. In this case,

$$
H(z+j b)=H(z), \quad K(z+j b)=K(z)
$$

for any natural number $)$.

Putting

from (3) $\mathrm{we}_{2}^{\pi}$ have

$$
\begin{aligned}
& h_{j}(z)=h(z+j b), \quad k_{j}(z)=k(z+j b), \\
& R_{j}(z)=R(z+j b), \quad p_{j}(z)=p(z+j b),
\end{aligned}
$$

$$
h_{j}-h=\left(k_{j}+K(z)\right) R_{j} e^{p_{\jmath}}-(k+K(z)) R e^{p},
$$


where $\jmath$ is a natural number. Hence

$$
\left(R_{\jmath} e^{p_{\jmath}}-R e^{p}\right) K(z)=\left(h_{j}-h\right)-\left(k_{j} R_{j} e^{p_{\jmath}}-k R e^{p}\right) .
$$

By using (24) for $\jmath=1,2$, and cancelling $K(z)$, we obtain the following identity

$$
\begin{aligned}
& R_{2}\left(h_{1}-h\right) e^{p_{2}}+R_{1} R_{2}\left(k_{2}-k_{1}\right) e^{p_{1}+p_{2}}-R R_{2}\left(k_{2}-k\right) e^{p+p_{2}} \\
+ & R R_{1}\left(k_{1}-k\right) e^{p+p_{1}}-R_{1}\left(h_{2}-h\right) e^{p_{1}}+R\left(h_{2}-h_{1}\right) e^{p}=0 .
\end{aligned}
$$

Dividing this relation by $\exp \left(p_{2}\right)$, we deduce

$$
\begin{aligned}
& R_{1} R_{2}\left(k_{2}-k_{1}\right) e^{p_{1}}-R R_{2}\left(k_{2}-k\right) e^{p}+R R_{1}\left(k_{1}-k\right) e^{p+p_{1}-p_{2}} \\
- & R_{1}\left(h_{2}-h\right) e^{p_{1}-p_{2}}+R\left(h_{2}-h_{1}\right) e^{p-p_{2}}=-R_{2}\left(h_{1}-h\right)(\equiv 0) .
\end{aligned}
$$

Assume

$$
p(z) \neq \text { constant }
$$

in which case, if we can prove

$$
p-p_{1}, \quad p-p_{2}, \quad p+p_{1}-p_{2} \neq \text { const., }
$$

then, applying Lemma A to the identity $(25)^{*}$, clearly we have a contradiction. We wish to prove that

$$
\text { when (26) holds, then } p-p_{\jmath} \neq \text { const., }
$$

for any natural number $\}$. Assume

$$
p_{\jmath}-p=\text { const. }=c, \text { or } e^{p_{\jmath}}=e^{c} e^{p},
$$

then (24) can be rewritten as

$$
K(z)=\frac{h_{j}-h}{e^{c} R_{j}-R} \cdot e^{-p}-\frac{e^{c} k_{j} R_{j}-k R}{e^{c} R_{j}-R},
$$

if

$$
e^{c} R_{j}-R \neq 0 .
$$

Then from (22), (29) and (30), using the notations (23), we have

$$
\begin{aligned}
& \frac{h_{j}-h}{e^{c} R_{j}-R}=e^{-c} \cdot \frac{h_{2 j}-h_{\jmath}}{e^{c} R_{2 \jmath}-R_{\jmath}}, \\
& \frac{e^{c} k_{\jmath} R_{\jmath}-k R}{e^{c} R_{\jmath}-R}=\frac{e^{c} k_{2 j} R_{2 j}-k_{j} R_{\jmath}}{e^{c} R_{2 j}-R_{\jmath}} .
\end{aligned}
$$

Hence by Lemma B, we have

$$
\begin{aligned}
& \frac{h_{\jmath}-h}{e^{c} R_{\jmath}-R}=\text { const. }=c^{\prime}(\neq 0), \\
& \frac{e^{c} k_{\jmath} R_{j}-k R}{e^{c} R_{\jmath}-R}=\text { const. }=c^{\prime \prime}(\neq 0),
\end{aligned}
$$

*) we'd multiply a common (entire) denominator, if necessary. 
or

$$
\left\{\begin{array}{l}
h_{j}-h=c^{\prime}\left(e^{c} R_{j}-R\right), \quad \text { and } \\
e^{c} k_{j} R_{j}-k R=c^{\prime \prime}\left(e^{c} R_{j}-R\right) .
\end{array}\right.
$$

Putting $R=v / u$ as in (4), and $R_{\jmath}=v_{j} / u_{\jmath}\left(u_{j}(z)=u(z+j b)\right.$, etc.), the upper relation of (32) reduces to

which is rewritten as

$$
c^{\prime}\left(e^{c} u v_{j}-u_{j} v\right)=u u_{j}\left(h_{j}-h\right)
$$

$$
-c^{\prime} \cdot \frac{u_{j} v}{u}=u_{j}\left(h_{j}-h\right)-c^{\prime} e^{c} v_{\jmath} .
$$

By using the quite similar argument around (18), we can conclude that $u(z)$ is constant. Hence we may assume $R(z)=v(z)$ without loss of generality, since $R=v / u$. Then the lower relation of (32) becomes

or

$$
e^{c} k_{j} v_{j}-k v=c^{\prime \prime}\left(e^{c} v_{j}-v\right) \text {, }
$$

$$
\left(c^{\prime \prime}-k\right) v=e^{c}\left(c^{\prime \prime}-k_{\jmath}\right) v_{\jmath} .
$$

Again by Lemma B, we conclude

which is impossible. Thus

$$
\left(c^{\prime \prime}-k\right) v=\text { const., }
$$

$$
e^{c} R,-R=0 \text { identically }
$$

is the only possibility remained. In this case, by Lemma $\mathrm{B}, R(z)$ is constant. Under (33), the identity (30) must reduce to

$$
\left(h_{j}-h\right) e^{-p}-\left(e^{c} k_{j} R_{j}-k R\right) \equiv 0 .
$$

But this will be clearly impossible, since $p(z)$ is non-constant and others $(h, k$ and $R, \cdots)$ are of order less than one $\left(h_{j}-h\right.$ cannot be identically zero). Thus we have proved the assertion (28).

Next assume

$$
p+p_{1}-p_{2}=\text { const. }=c \text {. }
$$

Then

$$
p_{1}-p_{2}=-p+c, \quad p-p_{2}=-p_{1}+c,
$$

hence (25) reduces to the following identity

$$
\begin{gathered}
R_{1} R_{2}\left(k_{2}-k_{1}\right) e^{p_{1}}-R R_{2}\left(k_{2}-k\right) e^{p}-e^{c} R_{1}\left(h_{2}-h\right) e^{-p} \\
+e^{c} R\left(h_{2}-h_{1}\right) e^{-p_{1}}+\left[e^{c} R R_{1}\left(k_{1}-k\right)+R_{2}\left(h_{1}-h\right)\right]=0 .
\end{gathered}
$$

Deviding this by $\exp \left(p_{1}\right)$, we obtain

$$
\begin{gathered}
-R R_{2}\left(k_{2}-k\right) e^{p-p_{1}}+\left[e^{c} R R_{1}\left(k_{1}-k\right)+R_{2}\left(h_{1}-h\right)\right] e^{-p_{1}} \\
-e^{c} R_{1}\left(h_{2}-h\right) e^{-p-p_{1}}+e^{c} R\left(h_{2}-h_{1}\right) e^{-2 p_{1}}=-R_{1} R_{2}\left(k_{2}-k_{1}\right) .
\end{gathered}
$$

Here, by (26) and (28) 
and

$$
p-p_{1}, \quad-p_{1}, \quad-2 p_{1} \neq \text { const., }
$$

$$
-R_{1} R_{2}\left(k_{2}-k_{1}\right) \not \equiv 0,
$$

since $k(z)$ is a non-constant entire function of order less than one. Also

Indeed if

$$
-p-p_{1}=-\left(p+p_{1}\right) \neq \text { const. }
$$

$$
p+p_{1}=\text { const. }=c
$$

then

$$
p_{1}+p_{2}=\text { const. }=c \text {. }
$$

Hence, subtracting (37) from (36), we get

$$
p-p_{2}=\text { const. }=0 \text {, }
$$

which contradicts the fact noted in (28).

Therefore we can apply Lemma A to the identity (35), and derive a contradiction. Hence (34) is impossible. proved.

Thus we have checked (27). Hence (26) is impossible,*) which is to be

4. In order to complete the proof of Theorem 1, by noting Propositions 1 and 2 , it is enough to show that the identity (3) is impossible to hold under the additional assumptions

$$
\begin{aligned}
& p(z) \text { is non-constant, and } \\
& b_{1} / b_{2} \text { is not a rational number } \\
& \left(b_{1} / b_{2}\right. \text { is a non-real complex number or } \\
& \text { real and irrational number). }
\end{aligned}
$$

Here we'll note the following fact, which is needed later.

Proposition 3. Let the identity (3) be valid, and assume the conditron (38). Then, for any natural number $\mathrm{J}$, the functions

$$
p\left(z+j b_{1}\right)-p(z) \text { and } p\left(z+j b_{2}\right)-p(z)
$$

are both non-constant.

Proof. Without loss of generality, it is sufficient to show

$$
p\left(z+j b_{1}\right)-p(z) \neq \text { const. }
$$

Indeed, for the proof of the fact

*) In this case, the fact that $R(z)$ 1s constant follows easily from (24) (without using Proposition 1). 


$$
p\left(z+j b_{2}\right)-p(z) \neq \text { const., }
$$

we need only to start the subsequent argument from the identity

$$
k(z)+K(z)=(h(z)+H(z)) \cdot 1 / R(z) \cdot e^{-p(z)} .
$$

We use the notations (10), (12), (14) and further

$$
q(z)=p\left(z+b_{2}\right), \quad q_{j}(z)=q\left(z+j b_{1}\right) .
$$

Assume (39) is not true, that is, for some natural number $\jmath$,

$$
p_{j}(z)-p(z)=\text { const. }=c \text {, }
$$

then also from (40) and (41)

$$
q_{j}(z)-q(z)=\text { const. }=c .
$$

Hence, noting $H\left(z+j b_{1}\right)=H(z)$, from (3) we have

$$
h_{j}-h=\left[e^{c}\left(k_{j}+K\left(z+j b_{1}\right)\right) R_{j}-(k+K(z)) R\right] e^{p},
$$

and noting $K\left(z+b_{2}\right)=K(z)$,

so that

$$
r_{j}-r=\left[e^{c}\left(s_{j}+K\left(z+j b_{1}\right)\right) S_{j}-(s+K(z)) S\right] e^{q},
$$

$$
\left\{\begin{array}{l}
R e^{p} K(z)-e^{c} R, e^{p} K\left(z+j b_{1}\right)=\left(e^{c} k_{j} R_{j}-k R\right) e^{p}-\left(h_{j}-h\right), \\
S e^{q} K(z)-e^{c} S_{j} e^{q} K\left(z+j b_{1}\right)=\left(e^{c} s_{\jmath} S_{j}-s S\right) e^{q}-\left(r_{j}-r\right) .
\end{array}\right.
$$

From the above system of equations, we get

$$
\left\{\begin{aligned}
& e^{p+q}\left(R S_{j}-R_{j} S\right) K(z)= S_{j} e^{q}\left[\left(e^{c} k_{j} R_{j}-k R\right) e^{p}-\left(h_{j}-h\right)\right] \\
&-R_{j} e^{p}\left[\left(e^{c} s_{j} S_{j}-s S\right) e^{q}-\left(r_{j}-r\right)\right], \\
& e^{p+q} e^{c}\left(R S_{j}-R, S\right) K\left(z+j b_{1}\right)= S e^{q}\left[\left(e^{c} k_{j} R_{j}-k R\right) e^{p}-\left(h_{j}-h\right)\right] \\
&-R e^{p}\left[\left(e^{c} s_{j} S_{j}-s S\right) e^{q}-\left(r_{j}-r\right)\right] .
\end{aligned}\right.
$$

Hence

$$
\begin{array}{r}
e^{p+q} e^{c}\left(R S_{j}-R_{\jmath} S\right)\left\{S_{2 j} e^{q_{j}}\left[\left(e^{c} k_{2 j} R_{2 j}-k_{j} R_{j}\right) e^{p_{\jmath}}-\left(h_{2 j}-h_{\jmath}\right)\right]\right. \\
\left.-R_{2 j} e^{p_{j}}\left[\left(e^{c} s_{2 j} S_{2 j}-s_{\jmath} S_{j}\right) e^{q_{\jmath}}-\left(r_{2 j}-r_{\jmath}\right)\right]\right\} \\
=e^{p_{\jmath}+q_{j}}\left(R_{\jmath} S_{2 j}-R_{2 j} S_{j}\right)\left\{S e^{q}\left[\left(e^{c} k_{j} R_{\jmath}-k R\right) e^{p}-\left(h_{j}-h\right)\right]\right. \\
- \\
\left.-R e^{p}\left[\left(e^{c} s_{\jmath} S_{j}-s S\right) e^{q}-\left(r_{j}-r\right)\right]\right\} .
\end{array}
$$

Using

$$
\begin{aligned}
& p+q+p_{j}+q_{j}=2 p+2 q+2 c, \\
& p+q+q_{\jmath}=p+2 q+c, \\
& p+q+p_{j}=2 p+q+c, \\
& p_{j}+q_{j}+q=p+2 q+2 c, \\
& p+p_{j}+q_{j}=2 p+q+2 c,
\end{aligned}
$$

we have the following identical relation 


$$
\begin{aligned}
& e^{2 c}\left[\left(R S_{\jmath}-R_{\jmath} S\right) R_{2 j}\left(r_{2 j}-r_{\jmath}\right)-\left(R_{\jmath} S_{2 j}-R_{2 \jmath} S_{j}\right) R\left(r_{j}-r\right)\right] e^{2 p+q} \\
& -e^{2 c}\left[\left(R S_{j}-R_{\jmath} S\right) S_{2 \jmath}\left(h_{2 j}-h_{\jmath}\right)-\left(R_{\jmath} S_{2 j}-R_{2 j} S_{j}\right) S\left(h_{j}-h\right)\right] e^{p+2 q} \\
& +e^{2 c}\left[e^{c}\left(R S_{\jmath}-R_{\jmath} S\right) S_{2 \jmath}\left(e^{c} k_{2 j} R_{2 j}-k_{j} R_{\jmath}\right)-e^{c}\left(R S_{j}-R_{\jmath} S\right) R_{2 \jmath}\left(e^{c} S_{2 \jmath} S_{2 \jmath}\right.\right. \\
& \left.\quad-s_{\jmath} S_{j}\right)-\left(R_{\jmath} S_{2 j}-R_{2 \jmath} S_{j}\right) S\left(e^{c} k_{j} R_{j}-k R\right) \\
& \left.\quad+\left(R_{\jmath} S_{2 \jmath}-R_{2 \jmath} S_{\jmath}\right) R\left(e^{c} s_{\jmath} S_{\jmath}-s S\right)\right] e^{2 p+2 q}=0
\end{aligned}
$$

Dividing the above identity by $e^{2 p+q}$ or $e^{p+2 q}$, and applying Lemma A, we conclude that three $[* * * * *]$ are all identically zero, since now because $\phi_{j}=p+c$ and $b_{1} / b_{2}$ is not a rational number, $p-q$ is non-constant.* $)_{1}$

From the fact that the first $[* * *]=0$ and the second $[* * *]=0$ we obtain

$$
\frac{R S_{j}-R_{j} S}{R\left(r_{j}-r\right)}=\frac{R_{j} S_{2 j}-R_{2 j} S_{j}}{R_{2 j}\left(r_{2 j}-r_{j}\right)}
$$

and

$$
\frac{R S_{j}-R_{\jmath} S}{S\left(h_{j}-h\right)}=\frac{R_{\jmath} S_{2 j}-R_{2 \jmath} S_{\jmath}}{S_{2 \jmath}\left(h_{2 \jmath}-h_{\jmath}\right)}
$$

Multiplying $1 / R$, to the both side of (42), by Lemma $\mathrm{B}$, we have

$$
\frac{R S_{j}-R_{\jmath} S}{R R_{\jmath}\left(r_{\jmath}-r\right)}=\text { const. }=c \text {. }
$$

Rewriting (44) as $S_{j} / R_{j}-S / R=c\left(r_{j}-r\right)$, we obtain

$$
S_{j} / R_{j}-c r_{j}=S / R-c r,
$$

whence, using Lemma B, we conclude

$$
S / R-c r=\text { const. }=c^{\prime}, \quad \text { or } S / R=c r+c^{\prime} .
$$

We note here that the constant $c$ in (44) is zero if and only if $R(z)$ is constant. Indeed if $c=0$, then from (44) we have $S_{j} / R_{\jmath}=S / R$. Lemma B leads us $S / R$ is constant, and so again we know that $R(z)$ is constant (cf. the notation (12)).

From (43), similarly we have

$$
\frac{R S_{j}-R, S}{S S_{\jmath}\left(h_{j}-h\right)}=\text { const. }=c^{\prime \prime}
$$

and hence $R / S-R_{j} / S_{j}=c^{\prime \prime}\left(h_{j}-h\right)$, so that as before we conclude

$$
R / S+c^{\prime \prime} h=\text { const. }=c^{\prime \prime \prime} \text {, or } R / S=-c^{\prime \prime} h+c^{\prime \prime \prime} .
$$

Here also $c^{\prime \prime}=0$ if and only if $R(z)$ is constant.

Now from (45) and (47), we know

$$
\left(c r+c^{\prime}\right)\left(-c^{\prime \prime} h+c^{\prime \prime \prime}\right) \equiv 1,
$$

which is possible only when $c=0$ and $c^{\prime \prime}=0$. Hence $R(z)$ must be constant.*) ${ }_{1}$

Thus we may assume

*) ${ }_{1}$ See the final remark (added in the proof). 


$$
R(z) \equiv 1 \text { and hence } S(z)=R\left(z+b_{z}\right) \equiv 1 .
$$

In this case, the system of equations becomes

Hence

$$
\left\{\begin{array}{l}
K(z)-e^{c} K\left(z+j b_{1}\right)=\left(e^{c} k_{j}-k\right)-\left(h_{j}-h\right) e^{-p}, \\
K(z)-e^{c} K\left(z+j b_{1}\right)=\left(e^{c} s_{j}-s\right)-\left(r_{j}-r\right) e^{-q} .
\end{array}\right.
$$

$$
\left(h_{j}-h\right) e^{-p}-\left(r_{j}-r\right) e^{-q}=\left(e^{c} k_{j}-k\right)-\left(e^{\mathrm{c}} s_{j}-s\right),
$$

so that we have the identity

$$
\left(r_{j}-r\right) e^{p-q}+\left[\left(e^{c} k_{j}-k\right)-\left(e^{c} s_{j}-s\right)\right] e^{p}=h_{j}-h .
$$

Here $h,-h \neq 0$ and $p \neq \equiv$ const. And also

$$
p-q \neq \text { const., } *)_{2}
$$

since now $p_{j}-p=$ const., and $b_{1} / b_{2}$ is not a rational number (non-constant function $p(z)$ cannot doubly periodic). So by Lemma A the identity (48) is impossible to hold, a contradiction. Hence the assertion (39) follows, which completes the proof of Prop. 3.

5. In this section, we wish to deduce the new identical relation starting the identity (3). We assume the condition (38) from now on.

We use the former notations (10), (12), (14) and (40). For example,

$$
\begin{aligned}
& h_{j}(z)=h\left(z+j b_{1}\right), \quad h_{j}(z)=k\left(z+j b_{1}\right), \\
& R_{j}(z)=R\left(z+j b_{1}\right), \quad p_{j}(z)=p\left(z+j b_{1}\right) .
\end{aligned}
$$

As $H\left(z+b_{1}\right)=H(z)$, from (3);

we have

$$
h(z)+H(z)=(k(z)+K(z)) R(z) e^{p(z)},
$$

Hence

$$
h_{1}-h=\left(k_{1}+K\left(z+b_{1}\right)\right) R_{1} e^{p_{1}}-(k+K(z)) R e^{p} .
$$

$$
R e^{p} K(z)-R_{1} e^{p_{1}} K\left(z+b_{1}\right)=\left(k_{1} R_{1} e^{p_{1}}-k R e^{p}\right)-\left(h_{1}-h\right) .
$$

And, noting $K\left(z+b_{2}\right)=K(z)$ and $r(z)=h\left(z+b_{2}\right)$ etc., from (49) we have

$$
S e^{q} K(z)-S_{1} e^{q_{1}} K\left(z+b_{1}\right)=\left(s_{1} S_{1} e^{q_{1}}-s S e^{q}\right)-\left(r_{1}-r\right) .
$$

Setting

$$
R=v / u, \text { and } S=y / x
$$

$\left(x(z)=u\left(z+b_{2}\right), \quad y(z)=v\left(z+b_{2}\right)\right)$ as in (4), the identities (49) and (50) can be rewritten as

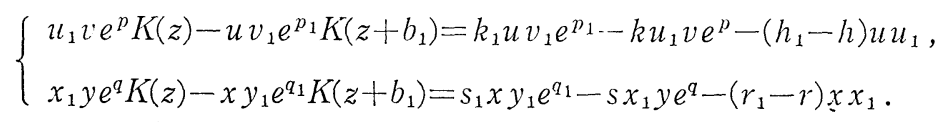

*) ${ }_{2}$ See the final remark (added in the proof). 
We solve this system of equations as follows:

$$
K(z)=D_{1}(z) / D(z), \quad K\left(z+b_{1}\right)=D_{2}(z) / D(z),
$$

where

$$
\begin{aligned}
& D(z)=\operatorname{det}\left(\begin{array}{ll}
u_{1} v e^{p}, & -u v_{1} e^{p_{1}} \\
x_{1} y e^{q}, & -x y_{1} e^{q_{1}}
\end{array}\right), \\
& D_{1}(z)=\operatorname{det}\left(\begin{array}{ll}
k_{1} u v_{1} e^{p_{1}}-k u_{1} v e^{p}-\left(h_{1}-h\right) u u_{1}, & -u v_{1} e^{p_{1}} \\
s_{1} x y_{1} e^{q_{1}}-s x_{1} y e^{q}-\left(r_{1}-r\right) x x_{1}, & -x y_{1} e^{q_{1}}
\end{array}\right), \\
& D_{2}(z)=\operatorname{det}\left(\begin{array}{ll}
u_{1} v e^{p}, & k_{1} u v_{1} e^{p_{1}}-k u_{1} v e^{p}-\left(h_{1}-h\right) u u_{1} \\
x_{1} y e^{q}, & s_{1} x y_{1} e^{q_{1}}-s x_{1} y e^{q}-\left(r_{1}-r\right) x x_{1}
\end{array}\right) .
\end{aligned}
$$

We note here that $D(z) \not \equiv 0$. Otherwise

$$
D(z)=x_{1} y u v_{1} e^{p_{1}+q}-x y_{1} u_{1} v e^{p+q_{1}} \equiv 0 .
$$

Then $\left(p+q_{1}\right)-\left(p_{1}+q\right)=$ const. $=c$ (say), or

$$
q_{1}=-p+p_{1}+q+c \text {. }
$$

If $D(z) \equiv 0$, then from (52) $D_{1}(z) \equiv 0$. This means

$$
\begin{aligned}
& k x y_{1} u_{1} v e^{p+q_{1}}+\left(s_{1}-k_{1}\right) x y_{1} u v_{1} e^{p_{1}+q_{1}} \\
- & s x_{1} y u v_{1} e^{p_{1}+q}+\left(h_{1}-h\right) x y_{1} u u_{1} e^{q_{1}} \\
- & \left(r_{1}-r\right) x x_{1} u v_{1} e^{p_{1}}=0 .
\end{aligned}
$$

From (55),

$$
p+q_{1}=p_{1}+q+c, \quad p_{1}+q_{1}=-p+2 p_{1}+q+c,
$$

so that (56) becomes

$$
\begin{aligned}
& \left(e^{c} k x y_{1} u_{1} v-s x_{1} y u v_{1}\right) e^{p_{1}+q}+e^{c}\left(s_{1}-k_{1}\right) x y_{1} u v_{1} e^{-p+2 p_{1}+q} \\
+ & e^{c}\left(h_{1}-h\right) x y_{1} u u_{1} e^{-p+p_{1}+q}-\left(r_{1}-r\right) x x_{1} u v_{1} e^{p_{1}}=0 .
\end{aligned}
$$

Dividing this by $\exp \left(-p+p_{1}+q\right)$, we obtain

$$
\begin{gathered}
\left(e^{c} k x y_{1} u_{1} v-s x_{1} y u v_{1}\right) e^{p}+e^{c}\left(s_{1}-k_{1}\right) x y_{1} u v_{1} e^{p_{1}} \\
-\left(r_{1}-r\right) x x_{1} u v_{1} e^{p-q}=-e^{c}\left(h_{1}-h\right) x y_{1} u u_{1}(\not \equiv 0) .
\end{gathered}
$$

Here $p, p_{1} \neq$ const., and also $p-q \neq$ const. by Prop. 3 (cf. (38)). Then applying Lemma A to (57), we have a contradiction. Thus

$$
D(z) \neq 0 \text {. }
$$

From (52), we know

$$
D(z) \cdot D_{1}\left(z+b_{1}\right) \equiv D\left(z+b_{1}\right) \cdot D_{2}(z) .
$$

Now

$$
D(z)=x_{1} y u v_{1} e^{p_{1}+q}-x y_{1} u_{1} v e^{p+q_{1}},
$$




$$
\begin{aligned}
& D\left(z+b_{1}\right)=x_{2} y_{1} u_{1} v_{2} e^{p_{2}+q_{1}}-x_{1} y_{2} u_{2} v_{1} e^{p_{1}+q_{2}}, \\
& D_{1}\left(z+b_{1}\right)=k_{1} x_{1} y_{2} u_{2} v_{1} e^{p_{1}+q_{2}}+\left(s_{2}-k_{2}\right) x_{1} y_{2} u_{1} v_{2} e^{p_{2}+q_{2}} \\
& -s_{1} x_{2} y_{1} u_{1} v_{2} e^{p_{2}+q_{1}}+\left(h_{2}-h_{1}\right) x_{1} y_{2} u_{1} u_{2} e^{q_{2}} \\
& -\left(r_{2}-r_{1}\right) x_{1} x_{2} u_{1} v_{2} e^{p_{2}} \text {, } \\
& D_{2}(z)=-(s-k) x_{1} y u_{1} v e^{p+q}+s_{1} x y_{1} u_{1} v e^{p+q_{1}} \\
& -k_{1} x_{1} y u v_{1} e^{p_{1}+q}-\left(r_{1}-r\right) x x_{1} u_{1} v e^{p} \\
& +\left(h_{1}-h\right) x_{1} y u u_{1} e^{q} \text {. }
\end{aligned}
$$

Hence from (59), we have the following identity after arrangement

$$
\begin{aligned}
& \left(r_{2}-r_{1}\right) X_{1} e^{p_{1}+p_{2}+q}+\left(r_{1}-r\right) X_{2} e^{p+p_{1}+q_{2}}+\left(h_{2}-h_{1}\right) X_{3} e^{p+q_{1}+q_{2}} \\
- & \left(r_{2}-r\right) X_{4} e^{p+p_{2}+q_{1}}-\left(h_{2}-h\right) X_{5} e^{p_{1}+q+q_{2}}+\left(h_{1}-h\right) X_{6} e^{p_{2}+q+q_{1}} \\
+ & (s-k) X_{7} e^{p+p_{1}+q+q_{2}}-(s-k) X_{8} e^{p+p_{2}+q+q_{1}}+\left(s_{2}-k_{2}\right) X_{9} e^{p+p_{2}+q_{1}+q_{2}} \\
- & \left(s_{1}-k_{1}\right) X_{10} e^{p+p_{1}+q_{1}+q_{2}}-\left(s_{2}-k_{2}\right) X_{11} e^{p_{1}+p_{2}+q+q_{2}} \\
+ & \left(s_{1}-k_{1}\right) X_{12} e^{p_{1}+p_{2}+q+q_{1}}=0 .
\end{aligned}
$$

Here we put

$$
\begin{array}{ll}
X_{1}=x_{1}{ }^{2} x_{2} y u u_{1} v_{1} v_{2}, & X_{2}=x x_{1}{ }^{2} y_{2} u_{1} u_{2} v v_{1}, \\
X_{3}=x x_{1} y_{1} y_{2} u_{1}{ }^{2} u_{2} v, & X_{1}=x x_{1} x_{2} y_{1} u_{1}^{2} v v_{2}, \\
X_{5}=x_{1}{ }^{2} y y_{2} u u_{1} u u_{2} v_{1}, & X_{6}=x_{1} x_{2} y y_{1} u u_{1}^{2} v_{2}, \\
X_{7}=x_{1}{ }^{2} y y_{2} u_{1} u_{2} v v_{1}, & X_{8}=x_{1} x_{2} y y_{1} u_{1}^{2} v v_{2}, \\
X_{9}=x x_{1} y_{1} y_{2} u_{1}{ }^{2} v v_{2}, & X_{10}=x x_{1} y_{1} y_{2} u_{1} u_{2} v v_{1}, \\
X_{11}=x_{1}^{2} y y_{2} u u_{1} v_{1} v_{2}, & X_{12}=x_{1} x_{2} y y_{1} u u_{1} v_{1} v_{2},
\end{array}
$$

each of which is an entire function and not identically zero such that the order is less than one.

Dividing the relation (60) by $\exp \left(p_{1}+p_{2}+q\right)$, we obtain

$$
\begin{aligned}
& \left(r_{2}-r_{1}\right) X_{1}+\left(r_{1}-r\right) X_{2} e^{p-p_{2}-q+q_{2}}+\left(h_{2}-h_{1}\right) X_{3} e^{p-p_{1}-p_{2}-q+q_{1}+q_{2}} \\
- & \left(r_{2}-r\right) X_{4} e^{p-p_{1}-q+q_{1}}-\left(h_{2}-h\right) X_{5} e^{-p_{2}+q_{2}}+\left(h_{1}-h\right) X_{6} e^{-p_{1}+q_{1}} \\
+ & (s-k) X_{7} e^{p-p_{2}+q_{2}}-(s-k) X_{8} e^{p-p_{1}+q_{1}}+\left(s_{2}-k_{2}\right) X_{9} e^{p-p_{1}-q+q_{1}+q_{2}} \\
- & \left(s_{1}-k_{1}\right) X_{10} e^{p-p_{2}-q+q_{1}+q_{2}}-\left(s_{2}-k_{2}\right) X_{11} e^{q_{2}}+\left(s_{1}-k_{1}\right) X_{12} e^{q_{1}}=0 .
\end{aligned}
$$

This is the new identity which we mention at the beginning of this section. In (62), we may transfer the term $\left(r_{2}-r_{1}\right) X_{1}$ to the right hand side if necessary.

6. In (62), as $r(z)=k\left(z+b_{2}\right)$ is a non-constant entire function of order less than one and $X_{1}$ is not identically zero, 


$$
\left(r_{2}-r_{1}\right) X_{1} \neq \equiv 0
$$

and as $p(z)$ is non-constant by assumption,

$$
q_{\jmath}(z)=p\left(z+j b_{1}+b_{2}\right) \not \equiv \text { const. }
$$

Also by Proposition 3, noting (40) etc., we know

$$
-p_{2}+q_{2}, \quad-p_{1}+q_{1} \neq \text { const., }
$$

and further, for any natural numbers $m$ and $n(m \neq n)$,

$$
\begin{aligned}
& p-p_{m}, \quad p+p_{m}, \quad p_{m}-p_{n}, \quad p_{m}+p_{n}, \\
& q-q_{m}, \quad q+q_{m}, \quad q_{m}-q_{n}, \quad q_{m}+q_{n}, \\
& p-q, \quad p+q, \quad p_{m}-q_{m}, \quad p_{m}+q_{m}
\end{aligned}
$$

are all non-constant by Proposition 3.

Indeed, for example if

$$
p+p_{m}=\text { const. }=c \text { (say), }
$$

then also

$$
p_{m}+p_{2 m}=\text { const. }=c,
$$

since $p_{j}(z)=p\left(z+j b_{1}\right)$. From (67) and (68), subtractıng,

or

$$
p_{2 m}-p=\text { const. }=0 \text {, }
$$

$$
p\left(z+2 m b_{1}\right)-p(z)=\text { const. }=0,
$$

which contradicts the fact proved in Proposition 3.

Also note that

$$
p-p_{1}-q+q_{1} \neq \text { const., if } p-p_{2}-q+q_{2} \neq \text { const. }
$$

Because, if

$$
p-p_{1}-q+q_{1}=\text { const. }=c \text { (say), }
$$

also, noting the notations,

$$
p_{1}-p_{2}-q_{1}+q_{2}=\text { const. }=c,
$$

so that, by adding (70) and (71), we have

$$
p-p_{2}-q+q_{2}=\text { const. }=2 c .
$$

Hence if we can prove the following six functions

$$
\begin{aligned}
& p-p_{2}-q+q_{2}, \quad p-p_{1}-p_{2}-q+q_{1}+q_{2}, \\
& p-p_{2}+q_{2}, \quad p-p_{1}+q_{1}, \\
& p-p_{1}-q+q_{1}+q_{2}, \quad p-p_{2}-q+q_{1}+q_{2}
\end{aligned}
$$

are non-constant, because of the facts (63), (64), (65) and (69), by applying Lemma 
A to the identity (62), we get a contradiction. Therefore the proof of Theorem 1 has become done.

In fact, in the following section, we'll prove that the six functions in (72) are all non-constant, one by one, applying Lemma A repeatedly.

7. The non-constancy of the six functions in (72).

[I] Non-constancy of $p-p_{2}-q+q_{2}$.

\section{Assume}

$$
p-p_{2}-q+q_{2}=\text { const. }=c .
$$

Then cancelling $q_{2}$, we have

$$
\begin{aligned}
& p-p_{1}-p_{2}-q+q_{1}+q_{2}=-p_{1}+q_{1}+c, \\
& -p_{2}+q_{2}=-p+q+c, \quad p-p_{2}+q_{2}=q+c, \\
& p-p_{1}-q+q_{1}+q_{2}=-p_{1}+p_{2}+q_{1}+c, \\
& p-p_{2}-q+q_{1}+q_{2}=q_{1}+c, \quad q_{2}=-p+p_{2}+q+c .
\end{aligned}
$$

Hence the identity (62) can be written as

$$
\begin{aligned}
& {\left[\left(r_{2}-r_{1}\right) X_{1}+\left(r_{1}-r\right) X_{2} e^{c}\right]+\left[\left(h_{2}-h_{1}\right) X_{3} e^{c}+\left(h_{1}-h\right) X_{6}\right] e^{-p_{1}+q_{1}} } \\
- & \left(r_{2}-r\right) X_{4} e^{p-p_{1}-q+q_{1}}-\left(h_{2}-h\right) X_{5} e^{c} e^{-p+q}+(s-k) X_{7} e^{c} e^{q} \\
- & (s-k) X_{8} e^{p-p_{1}+q_{1}}+\left(s_{2}-k_{2}\right) X_{9} e^{c} e^{-p_{1}+p_{2}+q_{1}} \\
- & \left(s_{1}-k_{1}\right)\left(X_{10} e^{c}-X_{12}\right) e^{q_{1}}-\left(s_{2}-k_{2}\right) X_{11} e^{c} e^{-p+p_{2}+q}=0 .
\end{aligned}
$$

Dividing this relation by $\exp \left(-p_{1}+p_{2}+q_{1}\right)$, we obtain the following identity.

$$
\begin{aligned}
& \left(s_{2}-k_{2}\right) X_{9} e^{c}+\left[\left(r_{2}-r_{1}\right) X_{1}+\left(r_{1}-r\right) X_{2} e^{c}\right] e^{p_{1}-p_{2}-q_{1}} \\
+ & {\left[\left(h_{2}-h_{1}\right) X_{3} e^{c}+\left(h_{1}-h\right) X_{6}\right] e^{-p_{2}}-\left(r_{2}-r\right) X_{4} e^{p-p_{2}-q} } \\
- & \left(h_{2}-h\right) X_{5} e^{c} e^{-p+p_{1}-p_{2}+q-q_{1}}+(s-k) X_{7} e^{c} e^{p_{1}-p_{2}+q-q_{1}} \\
- & (s-k) X_{8} e^{p-p_{2}}-\left(s_{1}-k_{1}\right)\left(X_{10} e^{c}-X_{12}\right) e^{p_{1}-p_{2}} \\
- & \left(s_{2}-k_{2}\right) X_{11} e^{c} e^{-p+p_{1}+q-q_{1}}=0 .
\end{aligned}
$$

Here, noting the notations (12) etc.,

$$
\begin{aligned}
& \left(s_{2}-k_{2}\right) X_{9} e^{c} \neq 0, \quad \text { and } \\
& -p_{2}, \quad p-p_{2}, \quad p_{1}-p_{2} \neq \text { const. }
\end{aligned}
$$

Further we shall prove that

$$
\begin{aligned}
& p_{1}-p_{2}-q_{1}, \quad p-p_{2}-q, \quad-p+p_{1}-p_{2}+q-q_{1}, \\
& p_{1}-p_{2}+q-q_{1}, \quad-p+p_{1}+q-q_{1}
\end{aligned}
$$

are non-constant, under (73). At first, we note that the former three are non- 
constant, and next we prove that the lower two are also non-constant.

Indeed if $p_{1}-p_{2}-q_{1}$ is constant, then by the notations given before, $p-p_{1}-q$ $=p_{1}-p_{2}-q_{1}=$ const. $=c^{\prime}$ (say). Adding these, $p-p_{2}-q-q_{1}=2 c^{\prime}$, which together with (73) show us that $q_{1}+q_{2}=$ const. $=c-2 c^{\prime}$. But this is contrary to the fact noted in (66).

If $p-p_{2}-q$ would be constant, then from (73) $q_{2}$ and hence $p(z)$ must be constant $\left(q_{2}(z) \equiv p\left(z+2 b_{1}+b_{2}\right)\right)$, a contradiction.

If $-p+p_{1}-p_{2}+q-q_{1}=$ const. $=c^{\prime}$, then $-p_{1}+p_{2}-p_{3}+q_{1}-q_{2}=c^{\prime}$ and so by adding we get $-p-p_{3}+q-q_{2}=$ const. $=2 c^{\prime}$ as above. Hence, using (73), we have $p_{2}+p_{3}=$ const. $=-\left(c+2 c^{\prime}\right)$. By (66) this is not valid, a contradiction.

\section{[I. 1] Assume}

$$
p_{1}-p_{2}+q-q_{1}=\text { const. }=c^{\prime} \text {. }
$$

Then, cancelling $q_{1}$,

$$
\begin{aligned}
& p_{1}-p_{2}-q_{1}=-q+c^{\prime}, \quad-p+p_{1}-p_{2}+q-q_{1}=-p+c^{\prime}, \\
& -p+p_{1}+q-q_{1}=-p+p_{2}+c^{\prime} .
\end{aligned}
$$

Hence (74) can be written as

$$
\begin{aligned}
& {\left[\left(s_{2}-k_{2}\right) X_{9}+(s-k) X_{7} e^{c^{\prime}}\right] e^{c}+\left[\left(r_{2}-r_{1}\right) X_{1}+\left(r_{1}-r\right) X_{2} e^{c}\right] e^{c^{\prime}} e^{-q} } \\
- & {\left[\left(h_{2}-h_{1}\right) X_{3} e^{c}+\left(h_{1}-h\right) X_{6}\right] e^{-p_{2}}-\left(r_{2}-r\right) X_{4} e^{p-p_{2}-q} } \\
- & \left(h_{2}-h\right) X_{5} e^{c+c^{\prime}} e^{-p}-(s-k) X_{8} e^{p-p_{2}}-\left(s_{1}-k_{1}\right)\left(X_{10} e^{c}-X_{12}\right) e^{p_{1}-p_{2}} \\
- & \left(s_{2}-k_{2}\right) X_{11} e^{c+c^{\prime}} e^{-p+p_{2}}=0 .
\end{aligned}
$$

Dividing this relation by $\exp \left(p-p_{2}\right)$, we obtain

Here

$$
\begin{aligned}
& -(s-k) X_{8}+\left[\left(s_{2}-k_{2}\right) X_{9}+(s-k) X_{\tau} e^{c^{\prime}}\right] e^{c} e^{-p+p_{2}} \\
+ & {\left[\left(r_{2}-r_{1}\right) X_{1}+\left(r_{1}-r\right) X_{2} e^{c}\right] e^{c^{\prime}} e^{-p+p_{2}-q} } \\
+ & {\left[\left(h_{2}-h_{1}\right) X_{3} e^{c}+\left(h_{1}-h\right) X_{6}\right] e^{-p}-\left(r_{2}-r\right) X_{4} e^{-q} } \\
- & \left(h_{2}-h\right) X_{5} e^{c+c^{\prime}} e^{-2 p+p_{2}}-\left(s_{1}-k_{1}\right)\left(X_{10} e^{c}-X_{12}\right) e^{-p+p_{1}} \\
- & \left(s_{2}-k_{2}\right) X_{11} e^{c+c^{\prime}} e^{-2\left(p-p_{2}\right)}=0 .
\end{aligned}
$$

$$
\begin{gathered}
-(s-k) X_{8} \neq 0, \quad \text { and } \\
-p+p_{2}, \quad-p, \quad-q, \quad-p+p_{1},-2\left(p-p_{2}\right) \neq \text { const. }
\end{gathered}
$$

Also we note that

$$
-p+p_{2}-q \text { and }-2 p+p_{2} \text { are non-constant. }
$$

Indeed if

$$
-p+p_{2}-q=\text { const. }=c^{\prime \prime} \text { (say) }
$$

then from (75) and (80), by adding, we have 
Again by adding

$$
\begin{aligned}
& -p+p_{1}-q_{1}=\text { const. }=c^{\prime}+c^{\prime \prime}, \text { and so } \\
& -p_{1}+p_{2}-q_{2}=c^{\prime}+c^{\prime \prime} .
\end{aligned}
$$

$$
-p+p_{2}-q_{1}-q_{2}=\text { const. }=2\left(c^{\prime}+c^{\prime \prime}\right),
$$

which together with (73) imply $q+q_{1}=$ const., contrary to (66). If

$$
-2 p+p_{2}=\text { const. }=c^{\prime \prime} \text { (say), }
$$

then in this case from (75) and (81)

$$
-2 p+p_{1}+q-q_{1}=-2 p_{1}+p_{2}+q_{1}-q_{2}=c^{\prime}+c^{\prime \prime},
$$

and hence, by adding,

$$
-2 p-p_{1}+p_{2}+q-q_{2}=\text { const. }=2\left(c^{\prime}+c^{\prime \prime}\right) \text {. }
$$

Using (73), from this we deduce $p+p_{1}=$ const., a contradiction.

As we have checked the facts (77), (78) and (79), applying Lemma $A$ to the identity (76), a contradiction follows. Thus (75) is impossible.

[I. 2] Assume now

$$
-p+p_{1}+q-q_{1}=\text { const. }=c^{\prime} \text { (say). }
$$

Then cancelling $q_{1}$,

$$
\begin{aligned}
& p_{1}-p_{2}-q_{1}=p-p_{2}-q+c^{\prime}, \quad-p+p_{1}-p_{2}+q-q_{1}=-p_{2}+c^{\prime}, \\
& p_{1}-p_{2}+q-q_{1}=p-p_{2}+c^{\prime} .
\end{aligned}
$$

Hence (74) can be written as

$$
\begin{aligned}
& \left(s_{2}-k_{2}\right) e^{c}\left(X_{9}-e^{c^{\prime}} X_{11}\right) \\
+ & {\left[\left\{\left(r_{2}-r_{1}\right) X_{1}+\left(r_{1}-r\right) X_{2} e^{c}\right\} e^{c^{\prime}}-\left(r_{2}-r\right) X_{4}\right] e^{p-p_{2}-q} } \\
+ & {\left[\left(h_{2}-h_{1}\right) X_{3} e^{c}+\left(h_{1}-h\right) X_{6}-\left(h_{2}-h\right) X_{5} e^{c+c^{\prime}}\right] e^{-p_{2}} } \\
+ & (s-k)\left(X_{7} e^{c+c^{\prime}}-X_{8}\right) e^{p-p_{2}}-\left(s_{1}-k_{1}\right)\left(X_{10} e^{c}-X_{12}\right) e^{p_{1}-p_{2}}=0 .
\end{aligned}
$$

Here

$$
\left(s_{2}-k_{2}\right) e^{c}\left(X_{9}-e^{c^{\prime}} X_{11}\right) \not \equiv 0 .
$$

If otherwise

$$
X_{9}-e^{c^{\prime}} X_{11} \equiv 0,
$$

since $s_{2}-k_{2} \neq 0$. (Note that $s_{2}(z)-k_{2}(z)=k\left(z+2 b_{1}+b_{2}\right)-k\left(z+2 b_{1}\right)$, by definition, and that $k(z)$ is a non-constant entire function of order less than one.)

Noting (82), (85) implies that

$$
X_{9} e^{p+q_{1}}-X_{11} e^{p_{1}+q} \equiv 0 .
$$

Recall 


$$
X_{9}=x x_{1} y_{1} y_{2} u_{1}^{2} v v_{2}, \quad X_{11}=x_{1}^{2} y y_{2} u u_{1} v_{1} v_{2}
$$

cf. (61). Then (86) reduces to

$$
x_{1} y_{2} u_{1} v_{2}\left(x y_{1} u_{1} v e^{p+q_{1}}-x_{1} y u v_{1} e^{p_{1}+q}\right) \equiv 0 .
$$

Since $x_{1} y_{2} u_{1} v_{2} \neq 00$, (87) shows

$$
D(z) \equiv 0
$$

(cf. (53)). However, in $\S 5$, we have ruled out the possibility (88). Thus (84) must be valid.

Now, $-p_{2}, p-p_{2}, p_{1}-p_{2} \neq$ const., and noting (73)

$$
p-p_{2}-q=c-q_{2}
$$

is also non-constant, since $q_{2}$ is so.

Then, under (84), by applying Lemma A to (83), we obtain a contradiction. Hence (82) is not valid.

Therefore, again, by applying Lemma A to the identity (74), we know that it cannot hold. This contradiction shows us that (73) is impossible. Thus the proof of the case $[I]$ is complete.

[II]. Non-constancy of $p-p_{1}-p_{2}-q+q_{1}+q_{2}$.

Assume

$$
p-p_{1}-p_{2}-q+q_{1}+q_{2}=\text { const. }=c .
$$

Then cancelling $q_{2}$,

$$
\begin{array}{ll}
p-p_{2}-q+q_{2}=p_{1}-q_{1}+c, & -p_{2}+q_{2}=-p+p_{1}+q-q_{1}+c, \\
p-p_{2}+q_{2}=p_{1}+q-q_{1}+c, & p-p_{1}-q+q_{1}+q_{2}=p_{2}+c, \\
p-p_{2}-q+q_{1}+q_{2}=p_{1}+c, & q_{2}=-p+p_{1}+p_{2}+q-q_{1}+c .
\end{array}
$$

Hence (62) can be written as

$$
\begin{aligned}
& {\left[\left(r_{2}-r_{1}\right) X_{1}+\left(h_{2}-h_{1}\right) X_{3} e^{c}\right]+\left(r_{1}-r\right) X_{2} e^{c} e^{p_{1}-q_{1}} } \\
- & \left(r_{2}-r\right) X_{4} e^{p-p_{1}-q+q_{1}}-\left(h_{2}-h\right) X_{5} e^{c} e^{-p+p_{1}+q-q_{1}}+\left(h_{1}-h\right) X_{6} e^{-p_{1}+q_{1}} \\
+ & (s-k) X_{7} e^{c} e^{p_{1}+q-q_{1}}-(s-k) X_{8} e^{p-p_{1}+q_{1}}+\left(s_{2}-k_{2}\right) X_{9} e^{c} e^{p_{2}} \\
- & \left(s_{1}-k_{1}\right) X_{10} e^{c} e^{p_{1}}-\left(s_{2}-k_{2}\right) X_{11} e^{c} e^{-p+p_{1}+p_{2}+q-q_{1}}+\left(s_{1}-k_{1}\right) X_{12} e^{q_{1}}=0 .
\end{aligned}
$$

Dividing this relation by $\exp \left(p_{1}-q_{1}\right)$, we have

$$
\begin{aligned}
& \left(r_{1}-r\right) X_{2} e^{c}+\left[\left(r_{2}-r_{1}\right) X_{1}+\left(h_{2}-h_{1}\right) X_{3} e^{c}\right] e^{-p_{1}+q_{1}} \\
- & \left(r_{2}-r\right) X_{4} e^{p-2 p_{1}-q+2 q_{1}}-\left(h_{2}-h\right) X_{5} e^{c} e^{-p+q}+\left(h_{1}-h\right) X_{6} e^{-2\left(p_{1}-q_{1}\right)} \\
+ & (s-k) X_{7} e^{c} e^{q}-(s-k) X_{8} e^{p-2 p_{1}+2 q_{1}}+\left(s_{2}-k_{2}\right) X_{9} e^{c} e^{-p_{1}+p_{2}+q_{1}} \\
- & \left(s_{1}-k_{1}\right) X_{10} e^{c} e^{q_{1}}-\left(s_{2}-k_{2}\right) X_{11} e^{c} e^{-p+p_{2}+q}+\left(s_{1}-k_{1}\right) X_{12} e^{-p_{1}+2 q_{1}}=0 .
\end{aligned}
$$

Here, 


$$
\begin{gathered}
\left(r_{1}-r\right) X_{2} e^{c} \neq 0, \\
-p_{1}+q_{1}, \quad-p+q, \quad-2\left(p_{1}-q_{1}\right), \quad q, \quad q_{1} \neq \text { const. }
\end{gathered}
$$

Next, under (89), we shall prove the non-constancy of the following five functions ;

$$
\begin{aligned}
& p-2 p_{1}-q+2 q_{1}, \quad p-2 p_{1}+2 q_{1},-p_{1}+p_{2}+q_{1}, \\
& -p+p_{2}+q, \quad-p_{1}+2 q_{1} .
\end{aligned}
$$

Then by Lemma A we have a contradiction from (90).

[II. 1]. If

$$
p-2 p_{1}-q+2 q_{1}=\text { const. }=c^{\prime} .
$$

Then $p_{1}-2 p_{2}-q_{1}+2 q_{2}=c^{\prime}$ also, and hence by adding, $p-p_{1}-2 p_{2}-q+q_{1}+2 q_{2}=2 c^{\prime}$.

This together with (89) imply that $p_{2}-q_{2}=$ const., which is a contradiction (cf. (66)).

[II. 2]. Assume

$$
p-2 p_{1}+2 q_{1}=\text { const. }=c^{\prime} \text {. }
$$

Then, changing the variable and adding, we have as before $p-p_{1}-2 p_{2}+2 q_{1}+2 q_{2}$ $=2 c^{\prime}$, whence by (89),

$$
p-p_{1}-2 q=\text { const. }=2 c-2 c^{\prime} .
$$

Now (92) and (93) give us that

$$
p_{1}-2 q-2 q_{1}=p_{2}-2 q_{1}-2 q_{2}=2 c-3 c^{\prime} .
$$

Also (93) and (94) give

$$
p-4 q-2 q_{1}=4 c-5 c^{\prime}
$$

Using (94) and (95), by cancelling $p, p_{1}$, and $p_{2}$,

$$
\begin{aligned}
& -p_{1}+q_{1}=-2 q-q_{1}-\left(2 c-3 c^{\prime}\right), \quad p-2 p_{1}-q+2 q_{1}=-q+c^{\prime}, \\
& -p+q=-3 q-2 q_{1}-\left(4 c-5 c^{\prime}\right), \quad-2 p_{1}+2 q_{1}=-4 q-2 q_{1}-2\left(2 c-3 c^{\prime}\right), \\
& -p_{1}+p_{2}+q_{1}=-2 q+q_{1}+2 q_{2}, \quad-p+p_{2}+q=-3 q+2 q_{2}-\left(2 c-2 c^{\prime}\right), \\
& -p_{1}+2 q_{1}=-2 q-\left(2 c-3 c^{\prime}\right) .
\end{aligned}
$$

Hence (90) reduces to

$$
\begin{aligned}
& {\left[\left(r_{1}-r\right) X_{2} e^{c}-(s-k) X_{8} e^{c^{\prime}}\right]+\left[\left(r_{2}-r_{1}\right) X_{1}+\left(h_{2}-h_{1}\right) X_{3} e^{c}\right] e^{-\left(2 c-3 c^{\prime}\right)} e^{-2 q-q_{1}}} \\
& -\left(r_{2}-r\right) X_{4} e^{c^{\prime}} e^{-q}-\left(h_{2}-h\right) X_{5} e^{c} e^{-\left(4 c-5 c^{\prime}\right)} e^{-3 q-2 q_{1}} \\
& +\left(h_{1}-h\right) X_{6} e^{-2\left(2 c-3 c^{\prime}\right)} e^{-4 q-2 q_{1}}+(s-k) X_{7} e^{c} e^{q} \\
& +\left(s_{2}-k_{2}\right) X_{9} e^{c} e^{-2 q+q_{1}+2 q_{2}}-\left(s_{1}-k_{1}\right) X_{10} e^{c} e^{q_{1}} \\
& -\left(s_{2}-k_{2}\right) X_{11} e^{c} e^{-\left(2 c-2 c^{\prime}\right)} e^{-3 q+2 q_{2}}+\left(s_{1}-k_{1}\right) X_{12} e^{-\left(2 c-3 c^{\prime}\right)} e^{-2 q}=0 .
\end{aligned}
$$


Dividing the above relation by $\exp (-q)$, we have

$$
\begin{aligned}
& -\left(r_{2}-r\right) X_{4} e^{c^{\prime}}+\left[\left(r_{1}-r\right) X_{2} e^{c}-(s-k) X_{8} e^{c^{\prime}}\right] e^{q} \\
+ & {\left[\left(r_{2}-r_{1}\right) X_{1}+\left(h_{2}-h_{1}\right) X_{3} e^{c}\right] e^{-\left(2 c-3 c^{\prime}\right)} e^{-q-q_{1}} } \\
- & \left(h_{2}-h\right) X_{5} e^{-3 c+5 c^{\prime}} e^{-2 q-2 q_{1}}+\left(h_{1}-h\right) X_{6} e^{-\left(2 c-3 c^{\prime}\right)} e^{-3 q-2 q_{1}} \\
+ & (s-k) X_{7} e^{c} e^{2 q}+\left(s_{2}-k_{2}\right) X_{9} e^{c} e^{-q+q_{1}+2 q_{2}}-\left(s_{1}-k_{1}\right) X_{10} e^{c} e^{q+q_{1}} \\
- & \left(s_{2}-k_{2}\right) X_{11} e^{-c+2 c^{\prime}} e^{-2 q+2 q_{2}}+\left(s_{1}-k_{1}\right) X_{12} e^{-\left(2 c-3 c^{\prime}\right)} e^{-q}=0 .
\end{aligned}
$$

Now, from (92) and (93) (changing the variable), we have

$$
\begin{aligned}
p-p_{1}-p_{2} & =\left(p-2 p_{1}+2 q_{1}\right)+\left(p_{1}-p_{2}-2 q_{1}\right) \\
& =\text { const. }=2 c-c^{\prime} .
\end{aligned}
$$

This together with (89) show

$$
q-q_{1}-q_{2}=\text { const. }=c-c^{\prime} .
$$

Using this fact, we can easily show that

$$
-3 q-2 q_{1}, \quad-q+q_{1}+2 q_{2} \neq \text { const. }
$$

Indeed if $-\left(3 q+2 q_{1}\right)=$ const. $=c^{\prime \prime}$ (say), then $-\left(3 q_{1}+2 q_{2}\right)=c^{\prime \prime}$ so that by (98) $2 q+q_{1}=2\left(q-q_{1}-q_{2}\right)+\left(3 q_{1}+2 q_{2}\right)$ becomes constant, and hence $q=2\left(2 q+q_{1}\right)-\left(3 q+2 q_{1}\right)$ must be so, a contradiction.

Also, $-q+q_{1}+2 q_{2}=-\left(q-q_{1}-q_{2}\right)+q_{2}$ cannot be constant by (98).

Further,

$$
\begin{gathered}
-\left(r_{2}-r\right) X_{4} e^{c^{\prime}} \neq 0, \\
q, \quad-q-q_{1}, \quad-2 q-2 q_{1}, \quad 2 q, \quad q+q_{1}, \quad-2 q+2 q_{2}, \quad-q \neq \text { const. }
\end{gathered}
$$

and, noting (99), by Lemma A, the identity (96) leads us to a contradiction. Thus (92) is impossible.

[II. 3]. Assume

$$
-p_{1}+p_{2}+q_{1}=\text { const. }=c^{\prime},
$$

and so,

$$
-p+p_{1}+q=c^{\prime}
$$

Then by subtracting (101) from (100), we have $p-2 p_{1}+p_{2}-q+q_{1}=$ const. $=0$. This and (89) give

$$
-p_{1}+2 p_{2}-q_{2}=-c \text { and hence }-p+2 p_{1}-q_{1}=-c \text {. }
$$

Then this and (100) give, by adding, $-p+p_{1}+p_{2}=-c+c^{\prime}$, so that by (89) we have

$$
q-q_{1}-q_{2}=\text { const. }=-c^{\prime} .
$$

While by (89) and (101), 


$$
p_{2}-q_{1}-q_{2}=p_{1}-q-q_{1}=\text { const. }=-c-c^{\prime} .
$$

Also by (101) and (103) we have

$$
p-2 q-q_{1}=\text { const. }=-c-2 c^{\prime} .
$$

By using (103) and (104), and cancelling $p, p_{1}$ and $p_{2}$, from (90) we deduce

$$
\begin{aligned}
& {\left[\left(r_{1}-r\right) X_{2}+\left(s_{2}-k_{2}\right) X_{9} e^{c^{\prime}}\right] e^{c}+\left[\left(r_{2}-r_{1}\right) X_{1}+\left(h_{2}-h_{1}\right) X_{3} e^{c}\right] e^{c+c^{\prime}} e^{-q} } \\
- & {\left[\left(r_{2}-r\right) X_{4}-\left(s_{1}-k_{1}\right) X_{12} e^{c^{\prime}}\right] e^{c} e^{-q+q_{1}} } \\
- & \left(h_{2}-h\right) X_{5} e^{2 c+2 c^{\prime}} e^{-q-q_{1}}+\left(h_{1}-h\right) X_{6} e^{2 c+2 c^{\prime}} e^{-2 q} \\
+ & (s-k) X_{7} e^{c} e^{q}-\left[(s-k) X_{8}+\left(s_{1}-k_{1}\right) X_{10}\right] e^{c} e^{q_{1}} \\
- & \left(s_{2}-k_{2}\right) X_{11} e^{c+c^{\prime}} e^{-q+q_{2}}=0 .
\end{aligned}
$$

Dividing this by $\exp (q)$, we have

$$
\begin{aligned}
& (s-k) X_{7} e^{c}+\left[\left(r_{1}-r\right) X_{2}+\left(s_{2}-k_{2}\right) X_{9} e^{c^{\prime}}\right] e^{c} e^{-q} \\
+ & {\left[\left(r_{2}-r_{1}\right) X_{1}+\left(h_{2}-h_{1}\right) X_{3} e^{c}\right] e^{c+c^{\prime}} e^{-2 q} } \\
- & {\left[\left(r_{2}-r\right) X_{4}-\left(s_{1}-k_{1}\right) X_{12} e^{c^{\prime}}\right] e^{c} e^{-2 q+q_{1}} } \\
- & \left(h_{2}-h\right) X_{5} e^{2 c+2 c^{\prime}} e^{-2 q-q_{1}}+\left(h_{1}-h\right) X_{6} e^{2 c+2 c^{\prime}} e^{-3 q} \\
- & {\left[(s-k) X_{8}+\left(s_{1}-k_{1}\right) X_{10}\right] e^{c} e^{-q+q_{1}}-\left(s_{2}-k_{2}\right) X_{11} e^{c+c^{\prime}} e^{-2 q+q_{2}}=0 . }
\end{aligned}
$$

Here

$$
\begin{gathered}
(s-k) X_{7} e^{c} \neq 0, \\
-q, \quad-2 q, \quad-3 q, \quad-q+q_{1} \neq \text { const. }
\end{gathered}
$$

Also, by using (102), we can easily show as before that

$$
-2 q+q_{1}, \quad-2 q-q_{1}, \quad-2 q+q_{2} \neq \text { const. }
$$

Hence, applying Lemma A to (105), we have that (100) is not valid.

[II. 4]. Assume

$$
-p+p_{2}+q=\text { const. }=c^{\prime} \text {. }
$$

Then, using (89), from (106) we have

and hence by (106),

$$
p_{1}-q_{1}-q_{2}=p-q-q_{1}=-c-c^{\prime} \text {, }
$$

$$
p_{2}-q_{1}=p_{1}-q=\text { const. }=-c .
$$

Again by (106) and (107)

$$
p-p_{1}-p_{2}=\text { const. }=c-c^{\prime} .
$$

Using (107), by cancelling $q$ and $q_{1}$, from (90) we deduce

$$
\begin{aligned}
& {\left[\left(r_{1}-r\right) X_{2}-\left(s_{2}-k_{2}\right) X_{11} e^{c}\right] e^{c}+\left[\left(r_{2}-r_{1}\right) X_{1}+\left(h_{2}-h_{1}\right) X_{3} e^{c}\right] e^{c} e^{-p_{1}+p_{2}} } \\
- & \left(r_{2}-r\right) X_{4} e^{c} e^{p-3 p_{1}+2 p_{2}}-\left(h_{2}-h\right) X_{5} e^{2 c} e^{-p+p_{1}}+\left(h_{1}-h\right) X_{6} e^{2 c} e^{-2 p_{1}+2 p_{2}}
\end{aligned}
$$




$$
\begin{aligned}
& +(s-k) X_{7} e^{2 c} e^{p_{1}}-(s-k) X_{8} e^{2 c} e^{p-2 p_{1}+2 p_{2}} \\
& +\left[\left(s_{2}-k_{2}\right) X_{9}+\left(s_{1}-k_{1}\right) X_{12}\right] e^{2 c} e^{-p_{1}+2 p_{2}} \\
& -\left(s_{1}-k_{1}\right) X_{10} e^{2 c} e^{p_{2}}=0 .
\end{aligned}
$$

Dividing this by $\exp \left(-p+p_{1}\right)$, we obtain

Here

$$
\begin{aligned}
& -\left(h_{2}-h\right) X_{5} e^{2 c}+\left[\left(r_{1}-r\right) X_{2}-\left(s_{2}-k_{2}\right) X_{11} e^{c}\right] e^{c} e^{p-p_{1}} \\
+ & {\left[\left(r_{2}-r_{1}\right) X_{1}+\left(h_{2}-h_{1}\right) X_{3} e^{c}\right] e^{c} e^{p-2 p_{1}+p_{2}} } \\
- & \left(r_{2}-r\right) X_{4} e^{c} e^{2\left(p-2 p_{1}+p_{2}\right)}+\left(h_{1}-h\right) X_{6} e^{2 c} e^{p-3 p_{1}+2 p_{2}} \\
+ & (s-k) X_{7} e^{2 c} e^{p}-(s-k) X_{8} e^{2 c} e^{2 p-3 p_{1}+2 p_{2}} \\
+ & {\left[\left(s_{2}-k_{2}\right) X_{9}+\left(s_{1}-k_{1}\right) X_{12}\right] e^{2 c} e^{p-2 p_{1}+2 p_{2}} } \\
- & \left(s_{1}-k_{1}\right) X_{10} e^{2 c} e^{p-p_{1}+p_{2}}=0 .
\end{aligned}
$$

$$
\begin{aligned}
& -\left(h_{2}-h\right) X_{5} e^{2 c} \neq 0, \\
& p-p_{1}, \quad p \neq \text { const. }
\end{aligned}
$$

Further, using (108), we can show arithmetically that

$$
\begin{aligned}
& p-2 p_{1}+p_{2}, \quad 2\left(p-2 p_{1}+p_{2}\right), \quad p-3 p_{1}+2 p_{2}, \\
& 2 p-3 p_{1}+2 p_{2}, \quad p-2 p_{1}+2 p_{2}, \quad p-p_{1}+p_{2} \neq \text { const. }
\end{aligned}
$$

Indeed, for example, if

$$
p-2 p_{1}+p_{2}=\text { const. }=c^{\prime \prime} \text { (say), }
$$

then, using (108), $2 p-3 p_{1}=$ const. $=c-c^{\prime}+c^{\prime \prime}$. Hence from (110) $2\left(p-2 p_{1}+p_{2}\right)-$ $\left(2 p-3 p_{1}\right)=-p_{1}+2 p_{2}=$ const., so that $-p+2 p_{1}=$ const. Then $p-p_{1}=\left(2 p-3 p_{1}\right)+$ $\left(-p+2 p_{1}\right)$ must become constant, which is impossible by (66). Thus $p-2 p_{1}+p_{2}$ $\neq$ const. Other cases are treated quite similarly.

Hence an application of Lemma A to (109) leads us to a contradiction Therefore (106) is impossible.

[II. 5]. Assume that

$$
-p_{1}+2 q_{1}=\text { const. }=c^{\prime}
$$

which implies that

$$
-p+2 q=-p_{2}+2 q_{2}=\text { const. }=c^{\prime} .
$$

Then by these, we have $-p+p_{1}+p_{2}+2 q-2 q_{1}-2 q_{2}=$ const. $=-c^{\prime}$, from which, together with (89), we have

$$
q-q_{1}-q_{2}=\text { const. }=c-c^{\prime} .
$$

Using (111) and (112), by cancelling $p, p_{1}$ and $p_{2}$, the identity (90) becomes

$$
\left[\left(r_{1}-r\right) X_{2} e^{c}+\left(s_{1}-k_{1}\right) X_{12} e^{c^{\prime}}\right]+\left[\left(r_{2}-r_{1}\right) X_{1}+\left(h_{2}-h_{1}\right) X_{3} e^{c}\right] e^{c^{\prime}} e^{-q_{1}}
$$




$$
\begin{aligned}
& -\left(r_{2}-r\right) X_{4} e^{c^{\prime}} e^{q-2 q_{1}}-\left(h_{2}-h\right) X_{5} e^{c} e^{c^{\prime}} e^{-q}+\left(h_{1}-h\right) X_{6} e^{2 c^{\prime}} e^{-2 q_{1}} \\
& +(s-k) X_{7} e^{c} e^{q}-(s-k) X_{8} e^{c^{\prime}} e^{2 q-2 q_{1}}+\left(s_{2}-k_{2}\right) X_{9} e^{c} e^{-q_{1}+2 q_{2}} \\
& -\left(s_{1}-k_{1}\right) X_{10} e^{c} e^{q_{1}}-\left(s_{2}-k_{2}\right) X_{11} e^{c} e^{-q+2 q_{2}}=0 .
\end{aligned}
$$

Dividing this identity by $\exp (-q)$, we deduce

Here

$$
\begin{aligned}
& -\left(h_{2}-h\right) X_{5} e^{c+c^{\prime}}+\left[\left(r_{1}-r\right) X_{2} e^{c}+\left(s_{1}-k_{1}\right) X_{12} e^{c^{\prime}}\right] e^{q} \\
+ & {\left[\left(r_{2}-r_{1}\right) X_{1}+\left(h_{2}-h_{1}\right) X_{3} e^{c}\right] e^{c^{\prime}} e^{q-q_{1}}-\left(r_{2}-r\right) X_{4} e^{c^{\prime}} e^{2\left(q-q_{1}\right)} } \\
+ & \left(h_{1}-h\right) X_{6} e^{2 c^{\prime}} e^{q-2 q_{1}}+(s-k) X_{7} e^{c} e^{2 q}-(s-k) X_{8} e^{c^{\prime}} e^{3 q-2 q_{1}} \\
+ & \left(s_{2}-k_{2}\right) X_{9} e^{c} e^{q-q_{1}+2 q_{2}}-\left(s_{1}-k_{1}\right) X_{10} e^{c} e^{q+q_{1}} \\
- & \left(s_{2}-k_{2}\right) X_{11} e^{c} e^{2 q_{2}}=0 .
\end{aligned}
$$

$$
\begin{gathered}
-\left(h_{2}-h_{1}\right) X_{5} e^{c+c^{\prime}} \neq 0, \\
q, \quad q-q_{1}, \quad 2\left(q-q_{1}\right), \quad 2 q, \quad q+q_{1}, \quad 2 q_{2} \neq \text { const. }
\end{gathered}
$$

Further, noting (113), we can easily show that

$$
q-2 q_{1}, \quad 3 q-2 q_{1}, \quad q-q_{1}+2 q_{2} \neq \text { const., }
$$

arithmetically, as before.

Hence, applying Lemma A to (114), we obtain that (111) is impossible.

Thus we've checked the assertion [II].

[III] Non-constancy of $p-p_{2}+q_{2}$.

Assume

$$
p-p_{2}+q_{2}=\text { const. }=c \text {. }
$$

Then cancelling $q_{2}$,

$$
\begin{aligned}
& p-p_{2}-q+q_{2}=-q+c, \quad p-p_{1}-p_{2}-q+q_{1}+q_{2}=-p_{1}-q+q_{1}+c, \\
& -p_{2}+q_{2}=-p+c, \quad p-p_{1}-q+q_{1}+q_{2}=-p_{1}+p_{2}-q+q_{1}+c, \\
& p-p_{2}-q+q_{1}+q_{2}=-q+q_{1}+c, \quad q_{2}=-p+p_{2}+c,
\end{aligned}
$$

so that $(62)$ can be rewritten as, after dividing the reduced relation by $\exp (-q)$,

$$
\begin{aligned}
& \left(r_{1}-r\right) X_{2} e^{c}+\left[\left(r_{2}-r_{1}\right) X_{1}+(s-k) X_{7} e^{c}\right] e^{q}+\left(h_{2}-h_{1}\right) X_{3} e^{c} e^{-p_{1}+q_{1}} \\
- & \left(r_{2}-r\right) X_{4} e^{p-p_{1}+q_{1}}-\left(h_{2}-h\right) X_{5} e^{c} e^{-p+q}+\left(h_{1}-h\right) X_{6} e^{-p_{1}+q+q_{1}} \\
- & (s-k) X_{8} e^{p-p_{1}+q+q_{1}}+\left(s_{2}-k_{2}\right) X_{9} e^{c} e^{-p_{1}+p_{2}+q_{1}} \\
- & \left(s_{1}-k_{1}\right) X_{10} e^{c} e^{q_{1}}-\left(s_{2}-k_{2}\right) X_{11} e^{c} e^{-p+p_{2}+q}+\left(s_{1}-k_{1}\right) X_{12} e^{q+q_{1}}=0 .
\end{aligned}
$$

Here, $\left(r_{1}-r\right) X_{2} e^{c} \equiv 0$, and that $q,-p_{1}+q_{1},-p+q, q_{1}$ and $q+q_{1}$ are non-constant. Also

$$
p-p_{1}+q_{1}, \quad-p_{1}+p_{2}+q_{1}, \quad-p+p_{2}+q \neq \text { const. }
$$


Indeed if $p-p_{1}+q_{1}$ is constant, then $p_{1}-p_{2}+q_{2}$ is so, which together with (115) give $p-p_{1}=$ const., a contradiction. If $-p_{1}+p_{2}+q_{1}$ is constant, then $-p_{2}+p_{3}+q_{2}$ is so. Then (115) shows $p-p_{3}=$ const., a contradiction. If $-p+p_{2}+q$ is constant, then (115) means $q+q_{2}=$ const., also a contradiction (cf. (66)).

Further we wish to prove that the following two functions

$$
-p_{1}+q+q_{1}, \quad p-p_{1}+q+q_{1}
$$

are non-constant under (115).

[III. 1]. Assume

$$
-p_{1}+q+q_{1}=\text { const. }=c^{\prime} \text {. }
$$

Then $-p_{2}+q_{1}+q_{2}=c^{\prime}$ also and hence by (115) we have

$$
p-q_{1}=\text { const. }=c-c^{\prime},
$$

which together with (117) give

$$
p-p_{1}+q=\text { const. }=c .
$$

Using (118) and (119), by cancelling $q$ and $q_{1}$ from (116), and then dividing the new identity by $\exp \left(p-p_{1}\right)$, we deduce

$$
\begin{aligned}
& \left(h_{2}-h_{1}\right) X_{3} e^{c^{\prime}}+\left[\left(r_{1}-r\right) X_{2} e^{c}+\left(h_{1}-h\right) X_{6} e^{c^{\prime}}\right] e^{-p+p_{1}} \\
+ & {\left[\left(r_{2}-r_{1}\right) X_{1}+(s-k) X_{7} e^{c}\right] e^{c} e^{-2\left(p-p_{1}\right)}-\left(r_{2}-r\right) X_{4} e^{-\left(c-c^{\prime}\right)} e^{p} } \\
- & \left(h_{2}-h\right) X_{5} e^{2 c} e^{-3 p+2 p_{1}}-\left[(s-k) X_{8}+\left(s_{1}-k_{1}\right) X_{10}\right] e^{c^{\prime}} e^{p_{1}} \\
- & \left(s_{2}-k_{2}\right) X_{9} e^{c^{\prime}} e^{p_{2}}-\left(s_{2}-k_{2}\right) X_{11} e^{2 c} e^{-3 p+2 p_{1}+p_{2}}+\left(s_{1}-k_{1}\right) X_{12} e^{c^{\prime}} e^{-p+2 p_{1}}=0 .
\end{aligned}
$$

Here $\left(h_{2}-h_{1}\right) X_{3} e^{c^{\prime}} \neq \equiv 0$, and that $-p+p_{1},-2\left(p-p_{1}\right), p, p_{1}$ and $p_{2}$ are non-constant. Now, from (119), $p_{1}-p_{2}+q_{1}=c$, which together with (118) give

$$
p+p_{1}-p_{2}=\text { const. }=2 c-c^{\prime} \text {. }
$$

Noting this fact (121), we can easily show that

$$
-3 p+2 p_{1}, \quad-3 p+2 p_{1}+p_{2}, \quad-p+2 p_{1} \neq \text { const. }
$$

Then, applying Lemma A, the identity (120) leads us to a contradiction. Hence (117) is impossible.

[III. 2]. Assume

$$
p-p_{1}+q+q_{1}=\text { const. }=c^{\prime} .
$$

Then $p_{1}-p_{2}+q_{1}+q_{2}=c^{\prime}$ also, with which and (115) we have

$$
p-p_{1}-q_{1}=c-c^{\prime} \text {. }
$$

Hence by (122)

$$
2 p-2 p_{1}+q=c \text {. }
$$

Using (123) and (124), by cancelling $q$ and $q_{1}$ from (116), and then dividing the 
rewritten identity by $\exp \left(-p+p_{1}\right)$, we obtain

$$
\begin{aligned}
& \left(s_{1}-k_{1}\right) X_{12} e^{c^{\prime}}+\left[\left(r_{1}-r\right) X_{2} e^{c}-(s-k) X_{8} e^{c^{\prime}}\right] e^{p-p_{1}} \\
+ & {\left[\left(r_{2}-r_{1}\right) X_{1}+(s-k) X_{7} e^{c}\right] e^{c} e^{-p+p_{1}}+\left(h_{2}-h_{1}\right) X_{3} e^{c^{\prime}} e^{2 p-3 p_{1}} } \\
- & \left(r_{2}-r\right) X_{4} e^{-c+c^{\prime}} e^{3\left(p-p_{1}\right)}-\left(h_{2}-h\right) X_{5} e^{2 c} e^{-2 p+p_{1}} \\
+ & \left(h_{1}-h\right) X_{6} e^{c^{\prime}} e^{-p_{1}}+\left(s_{2}-k_{2}\right) X_{9} e^{c^{\prime}} e^{2 p-3 p_{1}+p_{2}} \\
- & \left(s_{1}-k_{1}\right) X_{10} e^{c^{\prime}} e^{2\left(p-p_{1}\right)}-\left(s_{2}-k_{2}\right) X_{11} e^{2 c} e^{-2 p+p_{1}+p_{2}}=0 .
\end{aligned}
$$

Now from (123) and (124), we have

$$
p+p_{1}-2 p_{2}=\text { const. }=2 c-c^{\prime},
$$

since $2 p_{1}-2 p_{2}+q_{1}=c$ also by (124). Using (126), we can easily show that

$$
2 p-3 p_{1},-2 p+p_{1}, 2 p-3 p_{1}+p_{2} \text { and }-2 p+p_{1}+p_{2}
$$

are non-constant, as before. Further $\left(s_{1}-k_{1}\right) X_{12} e^{c^{\prime}} \not \equiv 0$, and that $p-p_{1}$ and $-p_{1}$ are non-constant. Hence applying Lemma A to (125), we deduce a contradiction. Thus (122) is not valid.

Therefore again applying Lemma A to the identity (116), we have a contradiction. Hence (115) is impossible, which is to be proved.

[IV]. Non-constancy of $p-p_{1}+q_{1}$.

Assume

$$
p-p_{1}+q_{1}=\text { const. }=c \text {. }
$$

Then,

$$
p_{1}-p_{2}+q_{2}=c \text { and } p-p_{2}+q_{1}+q_{2}=2 c \text {. }
$$

Hence cancelling $q_{1}$ and $q_{2}$, we have

$$
\begin{aligned}
& p-p_{2}-q+q_{2}=p-p_{1}-q+c, \quad p-p_{1}-p_{2}-q+q_{1}+q_{2}=-p_{1}-q+2 c, \\
& p-p_{1}-q+q_{1}=-q+c, \quad-p_{2}+q_{2}=-p_{1}+c, \quad-p_{1}+q_{1}=-p+c, \\
& p-p_{2}+q_{2}=p-p_{1}+c, \quad p-p_{1}-q+q_{1}+q_{2}=-p_{1}+p_{2}-q+2 c, \\
& p-p_{2}-q+q_{1}+q_{2}=-q+2 c, \quad q_{2}=-p_{1}+p_{2}+c, \quad q_{1}=-p+p_{1}+c .
\end{aligned}
$$

Hence the identity (62) reduces to the following one, after dividing the rewritten relation by $\exp \left(-p_{1}-q\right)$,

$$
\begin{aligned}
& \left(h_{2}-h_{1}\right) X_{3} e^{2 c}+\left[\left(r_{2}-r_{1}\right) X_{1}-(s-k) X_{8} e^{c}\right] e^{p_{1}+q} \\
+ & \left(r_{1}-r\right) X_{2} e^{c} e^{p}-\left[\left(r_{2}-r\right) X_{4}+\left(s_{1}-k_{1}\right) X_{10} e^{c}\right] e^{c} e^{p_{1}} \\
- & \left(h_{2}-h\right) X_{5} e^{c} e^{q}+\left(h_{1}-h\right) X_{6} e^{c} e^{-p+p_{1}+q}+(s-k) X_{\tau} e^{c} e^{p+q} \\
+ & \left(s_{2}-k_{2}\right) X_{9} e^{2 c} e^{p_{2}}-\left(s_{2}-k_{2}\right) X_{11} e^{c} e^{p_{2}+q}+\left(s_{1}-k_{1}\right) X_{12} e^{c} e^{-p+2 p_{1}+q}=0 .
\end{aligned}
$$

Here, $\left(h_{2}-h_{1}\right) X_{3} e^{2 c} \neq \equiv 0$, and that 


$$
p, \quad p_{1}, q,-p+p_{1}+q=-c+q+q_{1}, \quad p+q, \quad p_{2} \frac{1}{7} \text { const. }
$$

We must check the non-constancy of the following three functions

[IV. 1]. Assume

$$
p_{1}+q, \quad p_{2}+q, \quad-p+2 p_{1}+q .
$$

$$
p_{1}+q=\text { const. }=c^{\prime} \text {, }
$$

and hence $p_{2}+q_{1}=$ const. $=c^{\prime}$ also. Cancelling $q$ from (128) and then dividing the new identity by $\exp (p)$, we obtain

$$
\begin{aligned}
& \left(r_{1}-r\right) X_{2} e^{c}+\left[\left(h_{2}-h_{1}\right) X_{3} e^{2 c}+\left(r_{2}-r_{1}\right) X_{1} e^{c^{\prime}}-(s-k) X_{s} e^{c+c^{\prime}}\right] e^{-p} \\
- & {\left[\left(r_{2}-r\right) X_{4}+\left(s_{1}-k_{1}\right) X_{10} e^{c}\right] e^{c} e^{-p+p_{1}}-\left(h_{2}-h\right) X_{5} e^{c+c^{\prime}} e^{-p-p_{1}} } \\
+ & \left(h_{1}-h\right) X_{6} e^{c+c^{\prime}} e^{-2 p}+(s-k) X_{7} e^{c+c^{\prime}} e^{-p_{1}}+\left(s_{2}-k_{2}\right) X_{9} e^{2 c} e^{-p+p_{2}} \\
- & \left(s_{2}-k_{2}\right) X_{11} e^{c+c^{\prime}} e^{-p-p_{1}+p_{2}}+\left(s_{1}-k_{1}\right) X_{12} e^{c+c^{\prime}} e^{-2 p+p_{1}}=0 .
\end{aligned}
$$

Here, $-\left(r_{1}-r\right) X_{2} e^{c} \neq 0$, and that $-p,-p+p_{1},-p-p_{1},-2 p,-p_{1}$ and $-p+p_{2}$ are non-constant.

Now from (127) and (129) (since $p_{2}+q_{1}=c^{\prime}$ ), we have

$$
p-p_{1}-p_{2}=\text { const. }=c-c^{\prime} \text {. }
$$

Using (131), we can easily show that $-p-p_{1}+p_{2}$ and $-2 p+p_{1}$ are non-constant. Hence, applying Lemma A to (130), we get a contradiction. Thus (129) is impossible.

[IV. 2]. Assume

$$
p_{2}+q=\text { const. }=c^{\prime} \text {. }
$$

In this case, cancelling $q$ from (128) and then dividing by $\exp (\phi)$, we deduce the following identity.

$$
\begin{aligned}
& \left(r_{1}-r\right) X_{2} e^{c}+\left[\left(h_{2}-h_{1}\right) X_{3} e^{c}-\left(s_{2}-k_{2}\right) X_{11} e^{c^{\prime}}\right] e^{c} e^{-p} \\
+ & {\left[\left(r_{2}-r_{1}\right) X_{1}-(s-k) X_{8} e^{c}\right] e^{c^{\prime}} e^{-p+p_{1}-p_{2}} } \\
- & {\left[\left(r_{2}-r\right) X_{4}+\left(s_{1}-k_{1}\right) X_{10} e^{c}\right] e^{c} e^{-p+p_{1}} } \\
- & \left(h_{2}-h\right) X_{5} e^{c+c^{\prime}} e^{-p-p_{2}}+\left(h_{1}-h\right) X_{6} e^{c+c^{\prime}} e^{-2 p+p_{1}-p_{2}} \\
+ & (s-k) X_{7} e^{c+c^{\prime}} e^{-p_{2}}+\left(s_{2}-k_{2}\right) X_{9} e^{2 c} e^{-p+p_{2}} \\
+ & \left(s_{1}-k_{1}\right) X_{12} e^{c+c^{\prime}} e^{-2 p+2 p_{1}-p_{2}}=0 .
\end{aligned}
$$

Now from (132), $p_{3}+q_{1}=c^{\prime}$, from which and (127), we have

$$
p-p_{1}-p_{3}=\text { const. }=c-c^{\prime} \text {. }
$$

Using (134), we can easily show that

$$
-p+p_{1}-p_{2}, \quad-2 p+p_{1}-p_{2}, \quad-2 p+2 p_{1}-p_{2} \neq \text { const. }
$$

Indeed, for example, if 


$$
-2 p+2 p_{1}-p_{2}=\text { const., }
$$

then, using (134), by cancelling $p-p_{1}$, we get $p_{2}+2 p_{3}=$ const. Hence $p+2 p_{1}$ and $p_{1}+2 p_{2}$ are constant, so that, by subtracting these, we have

$$
p+p_{1}-2 p_{2}=\text { const. }
$$

Then from (135), cancelling $p_{2}$, we have $5 p-3 p_{1}=$ const. From this fact and $p+2 p_{1}=$ const., we conclude that $p=$ const., which is a contradiction. Thus (135) is impossible.

Further, clearly, $\left(r_{1}-r\right) X_{2} e^{c} \neq 0$, and that $-p,-p+p_{1},-p-p_{2},-p_{2}$ and $-p+p_{2}$ are non-constant. Hence applying Lemma A to (133), we conclude as before that (132) is not valid.

[IV. 3]. Assume

$$
-p+2 p_{1}+q=\text { const. }=c^{\prime} .
$$

Then, cancelling $q$ from (128) and then dividing by $\exp (p)$, we obtain

$$
\begin{aligned}
& \left(r_{1}-r\right) X_{2} e^{c}+\left[\left(h_{2}-h_{1}\right) X_{3} e^{c}+\left(s_{1}-k_{1}\right) X_{12} e^{c^{\prime}}\right] e^{c} e^{-p} \\
+ & {\left[\left(r_{2}-r_{1}\right) X_{1}-(s-k) X_{8} e^{c}\right] e^{c^{\prime}} e^{-p_{1}} } \\
- & {\left[\left(r_{2}-r\right) X_{4}+\left(s_{1}-k_{1}\right) X_{10} e^{c}\right] e^{c} e^{-p+p_{1}}-\left(h_{2}-h\right) X_{5} e^{c+c^{\prime}} e^{-2 p_{1}} } \\
+ & \left(h_{1}-h\right) X_{6} e^{c+c^{\prime}} e^{-p-p_{1}}+(s-k) X_{\tau} e^{c+c^{\prime}} e^{p-2 p_{1}} \\
+ & \left(s_{2}-k_{2}\right) X_{9} e^{2 c} e^{-p+p_{2}}-\left(s_{2}-k_{2}\right) X_{11} e^{c+c^{\prime}} e^{-2 p_{1}+p_{2}=0 .}
\end{aligned}
$$

Now from (136), $-p_{1}+2 p_{2}+q_{1}=c^{\prime}$ and hence by (127)

$$
p-2 p_{2}=\text { const. }=c-c^{\prime} \text {. }
$$

Using this, we can easily show that $p-2 p_{1}$ and $-2 p_{1}+p_{2}$ are non-constant. Other conditions are clearly satisfied. Hence, applying Lemma A to (137), we have that (136) is impossible.

Again applying Lemma A to (128), we obtain a contradiction. Hence (127) is not valid, which is to be proved.

[V]. Non-constancy of $p-p_{1}-q+q_{1}+q_{2}$.

Assume

$$
p-p_{1}-q+q_{1}+q_{2}=\text { const. }=c .
$$

Then, cancelling $\varphi_{2}$,

$$
\begin{aligned}
& p-p_{2}-q+q_{2}=p_{1}-p_{2}-q_{1}+c, \quad p-p_{1}-p_{2}-q+q_{1}+q_{2}=-p_{2}+c, \\
& -p_{2}+q_{2}=-p+p_{1}-p_{2}+q-q_{1}+c, \quad p-p_{2}+q_{2}=p_{1}-p_{2}+q-q_{1}+c, \\
& p-p_{2}-q+q_{1}+q_{2}=p_{1}-p_{2}+c, \quad q_{2}=-p+p_{1}+q-q_{1}+c .
\end{aligned}
$$

Hence the identity (62) becomes, after dividing by $\exp \left(-p_{2}\right)$

$$
\left(h_{2}-h_{1}\right) X_{3} e^{c}+\left[\left(r_{2}-r_{1}\right) X_{1}+\left(s_{2}-k_{2}\right) X_{9} e^{c}\right] e^{p_{2}}
$$


Here,

$$
\begin{aligned}
& +\left(r_{1}-r\right) X_{2} e^{c} e^{p_{1}-q_{1}}-\left(r_{2}-r\right) X_{4} e^{p-p_{1}+p_{2}-q+q_{1}} \\
& -\left(h_{2}-h\right) X_{5} e^{c} e^{-p+p_{1}+q-q_{1}}+\left(h_{1}-h\right) X_{6} e^{-p_{1}+p_{2}+q_{1}} \\
& +(s-k) X_{7} e^{c} e^{p_{1}+q-q_{1}}-(s-k) X_{8} e^{p-p_{1}+p_{2}+q_{1}} \\
& -\left(s_{1}-k_{1}\right) X_{10} e^{c} e^{p_{1}}-\left(s_{2}-k_{2}\right) X_{11} e^{c} e^{-p+p_{1}+p_{2}+q-q_{1}} \\
& +\left(s_{1}-k_{1}\right) X_{12} e^{p_{2}+q_{1}}=0 .
\end{aligned}
$$

$$
\begin{gathered}
\left(h_{2}-h_{1}\right) X_{3} e^{c} \neq 0, \\
p_{2}, \quad p_{1}-q_{1}, \quad p-p_{1}+p_{2}-q+q_{1}=c+p_{2}-q_{2}, \quad p_{1}, \\
-p+p_{1}+q-q_{1}=-c+q_{2}, \quad-p+p_{1}+p_{2}+q-q_{1}=-c+p_{2}+q_{2} \neq \text { const. }
\end{gathered}
$$

Also

$$
-p_{1}+p_{2}+q_{1} \neq \text { const., }
$$

since otherwise $-p+p_{1}+q$ is constant, which together with (138) give $q_{1}+q_{2}=$ const., a contradiction.

Further we must show that

$$
p_{1}+q-q_{1}, \quad p-p_{1}+p_{2}+q_{1}, \quad p_{2}+q_{1} \neq \text { const. }
$$

[V. 1]. Assume

$$
p_{1}+q-q_{1}=\text { const. }=c^{\prime} .
$$

Then $p_{2}+q_{1}-q_{2}=c^{\prime}$ also, and by adding these, $p_{1}+p_{2}+q-q_{2}=2 c^{\prime}$, which together with (138) give

$$
p+p_{2}+q_{1}=c+2 c^{\prime} .
$$

Hence from (140) and (141), we have

$$
p+p_{1}+p_{2}+q=\text { const. }=c+3 c^{\prime} .
$$

Using (141) and (142), by cancelling $q$ and $q_{1}$ from (139) and then dividing the rewritten relation by $\exp \left(-p_{1}\right)$, we obtain the following identity

Here

$$
\begin{aligned}
& -(s-k) X_{8} e^{c+2 c^{\prime}}+\left[\left(h_{2}-h_{1}\right) X_{3}+(s-k) X_{\tau} e^{c^{\prime}}\right] e^{c} e^{p_{1}} \\
+ & {\left[\left(r_{2}-r_{1}\right) X_{1}+\left(s_{2}-k_{2}\right) X_{9} e^{c}\right] e^{p_{1}+p_{2}}+\left(r_{1}-r\right) X_{2} e^{-2 c^{\prime}} e^{p+2 p_{1}+p_{2}} } \\
- & \left(r_{2}-r\right) X_{4} e^{-c^{\prime}} e^{p+p_{1}+p_{2}}-\left[\left(h_{2}-h\right) X_{5}-\left(s_{1}-k_{1}\right) X_{12} e^{c^{\prime}}\right] e^{c+c^{\prime}} e^{-p+p_{1}} \\
+ & \left(h_{1}-h\right) X_{6} e^{c+2 c^{\prime}} e^{-p}-\left(s_{1}-k_{1}\right) X_{10} e^{c} e^{2 p_{1}} \\
- & \left(s_{2}-k_{2}\right) X_{11} e^{c+c^{\prime}} e^{-p+p_{1}+p_{2}}=0 .
\end{aligned}
$$

$$
\begin{gathered}
-(s-k) X_{8} e^{c+2 c^{\prime}} \neq 0, \\
p_{1}, \quad p_{1}+p_{2}, \quad-p+p_{1}, \quad-p, \quad 2 p_{1} \neq \text { const. }
\end{gathered}
$$

Now note that from (141) and (142) we get 


$$
p-p_{1}-p_{3}=\text { const }=-c^{\prime} \text {. }
$$

Using this, we can show that

$$
p+2 p_{1}+p_{2}, \quad p+p_{1}+p_{2}, \quad-p+p_{1}+p_{2} \neq \text { const. }
$$

We check here the first case. Other two cases are quite easy. Assume

$$
p+2 p_{1}+p_{2}=\text { const. }=c^{\prime \prime} \text { (say). }
$$

Then also $p_{1}+2 p_{2}+p_{3}=c^{\prime \prime}$, which together with (144) give

$$
p+2 p_{2}=\text { const. }=-c^{\prime}+c^{\prime \prime} \text {. }
$$

From this and (145), by cancelling $p$, we have

$$
2 p_{1}-p_{2}=2 p-p_{1}=\text { const. }=c^{\prime} \text {. }
$$

Hence from the above two facts, we have that $p+4 p_{1}=\left(p+2 p_{2}\right)+2\left(2 p_{1}-p_{2}\right)$ is constant, so that $9 p=4\left(2 p-p_{1}\right)+\left(p+4 p_{1}\right)=$ const., a contradiction.

Hence applying Lemma A to (143), we conclude that (140) is impossible.

[V. 2]. Assume

$$
p-p_{1}+p_{2}+q_{1}=\text { const. }=c^{\prime} .
$$

Then $p_{1}-p_{2}+p_{3}+q_{2}=c^{\prime}$ also and hence by adding these

$$
p+p_{3}+q_{1}+q_{2}=\text { const. }=2 c^{\prime} \text {. }
$$

From this and (138) we have

$$
p_{1}+p_{3}+q=\text { const. }=-c+2 c^{\prime} \text {. }
$$

Note that from (146) and (147) we get

$$
p-p_{1}-p_{4}=\text { const. }=c-c^{\prime},
$$

since $p_{2}+p_{4}+q_{1}=-c+2 c^{\prime}$ by $(147)$.

Using (146) and (147), by cancelling $q$ and $q_{1}$ from (139) and then dividing by $\exp \left(p_{1}\right)$, we obtain

$$
\begin{aligned}
& -\left(s_{1}-k_{1}\right) X_{10} e^{c}+\left[\left(h_{2}-h_{1}\right) X_{3} e^{c}-(s-k) X_{8} e^{c^{\prime}}\right] e^{-p_{1}} \\
+ & {\left[\left(r_{2}-r_{1}\right) X_{1}+\left(s_{2}-k_{2}\right) X_{9} e^{c}\right] e^{-p_{1}+p_{2}}+\left(r_{1}-r\right) X_{2} e^{c-c^{\prime}} e^{p-p_{1}+p_{2}} } \\
- & \left(r_{2}-r\right) X_{4} e^{c-c^{\prime}} e^{p_{3}}-\left(h_{2}-h\right) X_{5} e^{c^{\prime}} \cdot e^{-2 p_{1}+p_{2}-p_{3}} \\
+ & \left(h_{1}-h\right) X_{6} e^{c^{\prime}} e^{-p-p_{1}}+(s-k) X_{7} e^{c^{\prime}} \cdot e^{p-2 p_{1}+p_{2}-p_{3}} \\
- & \left(s_{2}-k_{2}\right) X_{11} e^{c^{\prime}} \cdot e^{-2 p_{1}+2 p_{2}-p_{3}}+\left(s_{1}-k_{1}\right) X_{12} e^{c^{\prime}} e^{-p}=0 .
\end{aligned}
$$

Here $-\left(s_{1}-k_{1}\right) X_{10} e^{c} \not \equiv 0$ and that $-p_{1},-p_{1}+p_{2}, p_{3},-p-p_{1}$ and $-p$ are nonconstant. Further, using (148), we can easily prove that

$$
p-p_{1}+p_{2}, \quad-2 p_{1}+p_{2}-p_{3}, \quad p-2 p_{1}+p_{2}-p_{3} \text { and }-2 p_{1}+2 p_{2}-p_{3}
$$

are non-constant. Hence applying Lemma A to (149), we can conclude that (146) 
is impossible.

[V. 3]. Assume

$$
p_{2}+q_{1}=\text { const. }=c^{\prime} \text {. }
$$

Then also

$$
p_{1}+q=p_{3}+q_{2}=\text { const. }=c^{\prime} .
$$

From (150) and (151),

$$
p_{1}-p_{2}-p_{3}+q-q_{1}-q_{2}=\text { const. }=-c^{\prime},
$$

and hence by (138) we have

$$
p-p_{2}-p_{3}=\text { const. }=c-c^{\prime} .
$$

Using (150) and (151), by cancelling $q$ and $q_{1}$ from (139) and then dividing by $\exp (p)$, we deduce

$$
\begin{aligned}
& -\left(r_{2}-r\right) X_{4}+\left[\left(h_{2}-h_{1}\right) X_{3} e^{c}+\left(s_{1}-k_{1}\right) X_{12} e^{c^{\prime}}\right] e^{-p} \\
+ & {\left[\left(r_{2}-r_{1}\right) X_{1}+\left(s_{2}-k_{2}\right) X_{9} e^{c}+(s-k) X_{7} e^{c}\right] e^{-p+p_{2}} } \\
+ & \left(r_{1}-r\right) X_{2} e^{c-c^{\prime}} e^{-p+p_{1}+p_{2}}-\left(h_{2}-h\right) X_{5} e^{c} e^{-2 p+p_{2}} \\
+ & \left(h_{1}-h\right) X_{6} e^{c^{\prime}} e^{-p-p_{1}}-(s-k) X_{8} e^{c^{\prime}} e^{-p_{1}} \\
- & \left(s_{1}-k_{1}\right) X_{10} e^{c} e^{-p+p_{1}}-\left(s_{2}-k_{2}\right) X_{11} e^{c} e^{-2 p++p_{1}}=0 .
\end{aligned}
$$

Here $-\left(r_{2}-r\right) X_{1} \not \equiv 0$ and that $-p,-p+p_{2},-p-p_{1},-p_{1},-p+p_{1}$ and $-2\left(p-p_{2}\right)$ are non-constant. Further, using (152), we can easily show that

$$
-p+p_{1}+p_{2} \text { and }-2 p+p_{2}
$$

are non-constant. Hence applying Lemma A to (153) we get a contradiction, which shows that (150) is impossible.

Therefore again applying Lemma A to the identity (139), we obtain a contradiction. Thus (138) is not valid.

[VI]. Non-constancy of $p-p_{2}-q+q_{1}+q_{2}$.

Assume

$$
p-p_{2}-q+q_{1}+q_{2}=\text { const. }=c \text {. }
$$

Then cancelling $q_{2}$,

$$
\begin{aligned}
& p-p_{2}-q+q_{2}=-q_{1}+c, \quad p-p_{1}-p_{2}-q+q_{1}+q_{2}=-p_{1}+c, \\
& -p_{2}+q_{2}=-p+q-q_{1}+c, \quad p-p_{2}+q_{2}=q-q_{1}+c, \\
& p-p_{1}-q+q_{1}+q_{2}=-p_{1}+p_{2}+c, \quad q_{2}=-p+p_{2}+q-q_{1}+c .
\end{aligned}
$$

In this case, using the above facts and then dividing by $\exp \left(-q_{1}\right)$, from (62) we obtain the following identity

$$
\left(r_{1}-r\right) X_{2} e^{c}+\left[\left(r_{2}-r_{1}\right) X_{1}-\left(s_{1}-k_{1}\right) X_{10} e^{c}\right] e^{q_{1}}
$$


ON A CHARACTERISTIC PROPERTY OF PERIODIC ENTIRE FUNCTIONS

$$
\begin{aligned}
& +\left(h_{2}-h_{1}\right) X_{3} e^{c} e^{-p_{1}+q_{1}}-\left(r_{2}-r\right) X_{4} e^{p-p_{1}-q+2 q_{1}} \\
& -\left(h_{2}-h\right) X_{5} e^{c} e^{-p+q}+\left(h_{1}-h\right) X_{6} e^{-p_{1}+2 q_{1}}+(s-k) X_{7} e^{c} e^{q} \\
& -(s-k) X_{8} e^{p-p_{1}+2 q_{1}}+\left(s_{2}-k_{2}\right) X_{9} e^{c} e^{-p_{1}+p_{2}+q_{1}} \\
& -\left(s_{2}-k_{2}\right) X_{11} e^{c} e^{-p+p_{2}+q}+\left(s_{1}-k_{1}\right) X_{12} e^{2 q_{1}}=0 .
\end{aligned}
$$

Here $\left(r_{1}-r\right) X_{2} e^{c} \neq 0$, and that $q_{1},-p_{1}+q_{1},-p+q, q$ and $-p+p_{2}+q_{1}=q_{1}+q_{2}-c$ are non-constant. Also

since otherwise

$$
p-p_{1}-q+2 q_{1} \neq \text { const., }
$$

$$
p-p_{2}-q+q_{1}+2 q_{2}=\left(p-p_{1}-q+2 q_{1}\right)+\left(p_{1}-p_{2}-q_{1}+2 q_{2}\right)
$$

is constant, and hence from (154) $q_{2}$ becomes so, which is a contradiction.

Subsequently, we'll prove that the following three functions

$$
-p_{1}+2 q_{1}, \quad p-p_{1}+2 q_{1}, \quad-p_{1}+p_{2}+q_{1}
$$

are non-constant.

[VI. 1]. Assume

$$
-p_{1}+2 q_{1}=\text { const. }=c^{\prime}
$$

Then also

$$
-p+2 q=-p_{2}+2 q_{2}=\text { const. }=c^{\prime} \text {. }
$$

From (157), $p-p_{2}-2 q+2 q_{2}=$ const. $=0$, and hence by (154),

$$
q+q_{1}-q_{2}=\text { const. }=c \text {. }
$$

Using (156) and (157), by cancelling $p, p_{1}$ and $p_{2}$ from (155), and then dividing by $\exp \left(-q_{1}\right)$, we obtain

$$
\begin{aligned}
& \left(h_{2}-h_{1}\right) X_{3} e^{c+c^{\prime}}+\left[\left(r_{1}-r\right) X_{2} e^{c}+\left(h_{1}-h\right) X_{6} e^{c^{\prime}}\right] e^{q_{1}} \\
+ & {\left[\left(r_{2}-r_{1}\right) X_{1}-\left(s_{1}-k_{1}\right) X_{10} e^{c}\right] e^{2 q_{1}} } \\
- & {\left[\left(r_{2}-r\right) X_{4}-(s-k) X_{7} e^{c}\right] e^{q+q_{1}}-\left(h_{2}-h\right) X_{5} e^{c+c^{\prime}} e^{-q+q_{1}} } \\
- & (s-k) X_{8} e^{2 q+q_{1}}+\left(s_{2}-k_{2}\right) X_{9} e^{c} e^{2 q_{2}}-\left(s_{2}-k_{2}\right) X_{11} e^{c} e^{-q+q_{1}+2 q_{2}} \\
+ & \left(s_{1}-k_{1}\right) X_{12} e^{3 q_{1}}=0 .
\end{aligned}
$$

Since, using (158), we can easily show that

$$
2 q+q_{1} \text { and }-q+q_{1}+2 q_{2}
$$

are non-constant, by applying Lemma A to (159) we have a contradiction. Hence (156) is impossible.

[VI. 2]. Assume

$$
p-p_{1}+2 q_{1}=\text { const. }=c^{\prime} .
$$

Then, $p_{1}-p_{2}+2 q_{2}=c^{\prime}$ also and hence 


$$
p-p_{2}+2 q_{1}+2 q_{2}=\text { const. }=2 c^{\prime} .
$$

Now from (154) and (161), we obtain

$$
q+q_{1}+q_{2}=\text { const. }=-c+2 c^{\prime} .
$$

Then, from (161) and (162), cancelling $q_{1}+q_{2}$, we have

and so

$$
p-p_{2}-2 q=\text { const. }=2 c-2 c^{\prime},
$$

$$
p_{1}-p_{3}-2 q_{1}=\text { const. }=2 c-2 c^{\prime} .
$$

From (160) and (163), we get

$$
p-p_{3}=\text { const. }=2 c-c^{\prime},
$$

which is contrary to the fact noted in (66). Thus (160) is not valid.

[VI. 3]. Assume

$$
-p_{1}+p_{2}+q_{1}=\text { const. }=c^{\prime} .
$$

Then also

$$
-p+p_{1}+q=\text { const. }=c^{\prime}
$$

and so by adding (164) and (165)

$$
-p+p_{2}+q+q_{1}=\text { const. }=2 c^{\prime} .
$$

From (154) and (166), we conclude

$$
2 q_{1}+q_{2}=2 q+q_{1}=\text { const. }=c+2 c^{\prime} .
$$

Also from (164) and (165) we have

$$
\begin{aligned}
-2 p+p_{1}+p_{2}+2 q+q_{1} & =2\left(-p+p_{1}+q\right)+\left(-p_{1}+p_{2}+q_{1}\right) \\
& =\text { const. }=3 c^{\prime} .
\end{aligned}
$$

Then by (167) and (168), we obtain

$$
-2 p+p_{1}+p_{2}=\text { const. }=-c+c^{\prime} .
$$

Using (164) and (165), by cancelling $q$ and $q_{1}$ from (155) and then dividing by $\exp \left(-p_{1}+p_{2}\right)$, we have

$$
\begin{aligned}
& -\left(s_{2}-k_{2}\right) X_{11} e^{c+c^{\prime}}+\left[\left(r_{1}-r\right) X_{2}+\left(s_{2}-k_{2}\right) X_{9} e^{c^{\prime}}\right] e^{c} e^{p_{1}-p_{2}} \\
+ & {\left[\left(r_{2}-r_{1}\right) X_{1}-\left(s_{1}-k_{1}\right) X_{10} e^{c}\right] e^{c^{\prime}} e^{2\left(p_{1}-p_{2}\right\rangle} } \\
+ & \left(h_{2}-h_{1}\right) X_{3} e^{c+c^{\prime}} e^{p_{1}-2 p_{2}}-\left[\left(r_{2}-r\right) X_{4}-\left(s_{1}-k_{1}\right) X_{12} e^{c^{\prime}}\right] e^{c^{\prime}} e^{3\left(p_{1}-p_{2}\right)} \\
- & \left(h_{2}-h\right) X_{5} e^{c+c^{\prime}} e^{-p_{2}}+\left(h_{1}-h\right) X_{6} e^{2 c^{\prime}} e^{2 p_{1}-3 p_{2}} \\
+ & (s-k) X_{7} e^{c+c^{\prime}} e^{p-p_{2}}-(s-k) X_{8} e^{2 c^{\prime}} e^{p+2 p_{1}-3 p_{2}}=0 .
\end{aligned}
$$

Here,

$$
-\left(s_{2}-k_{2}\right) X_{11} e^{c+c^{\prime}} \neq 0 \text {, }
$$




$$
p_{1}-p_{2}, 2\left(p_{1}-p_{2}\right), \quad 3\left(p_{1}-p_{2}\right), \quad-p_{2}, \quad p-p_{2} \neq \text { const. }
$$

Further, using (169), we can easily show that

$$
p_{1}-2 p_{2}, 2 p_{1}-3 p_{2} \text { and } p+2 p_{1}-3 p_{2}
$$

are non-constant. Hence, by applying Lemma A to (170), we have a contradiction. This implies that (164) is impossible.

Again, applying Lemma A to (155), we have a contradiction, so that (154) is not valid.

We have checked the facts [I], [II], [III], [IV], [V] and [VI] as above. Therefore the proof of Theorem 1 is now complete.

\section{Proof of Theorem 2.}

By assumption, we have the following identical relation

$$
z-H_{1}(z)=\left(z-H_{2}(z)\right) R(z) e^{p(z)},
$$

where $R(z)$ is a meromorphic function $(\not \equiv 0)$ of order less than one and $p(z)$ is an entire function as in (3).

By Theorem $1, R(z)$ and $p(z)$ are constant and that $b_{1} / b_{2}$ is a rational number. Hence (171) reduces to

$$
z-H_{1}(z)=c \cdot\left(z-H_{2}(z)\right),
$$

where $c$ is a non-zero constant. We rewrite (172) as

$$
(1-c) z=H_{1}(z)-c \cdot H_{2}(z) \text {. }
$$

Since $b_{1} / b_{2}$ is a rational number, $m b_{1}=n b_{2}$ (=b, say) for some non-zero integers $m$ and $n$, so that the right hand side of (173) is periodic with period $b$. Hence we conclude that $c=1$. Then the identity (173) implies that

$$
H_{1}(z) \equiv H_{2}(z) \text {. }
$$

Thus the assertion of Theorem 2 follows.

\section{Remark.}

Our results can be generalized to the case of entire functions in several complex variables. For example, for the non-zero constants $b$ and $b^{\prime}$, we consider the following class

$$
G\left(b, b^{\prime}\right) \equiv\left\{F(z, w)=f(z)+g(w) ; f(z) \in G(b) \text { and } g(z) \in G\left(b^{\prime}\right)\right\} .
$$

Then we obtain from Theorem 1 that, when $F(z, w) \in G\left(b_{1}, b_{1}{ }^{\prime}\right)$ and $E(z, w) \in$ $G\left(b_{2}, b_{2}{ }^{\prime}\right)$ for some non-zero constants $b_{\jmath}, b_{\jmath}{ }^{\prime}(\jmath=1,2)$, if further the following identical relation

$$
F(z, w)=E(z, w) e^{p(z, w)}
$$


holds for some entire function $p(z, w)$ in two complex variables $z$ and $u$, then $p(z, w)$ must be constant and that $b_{1} / b_{2}$ and $b_{1}{ }^{\prime} / b_{2}{ }^{\prime}$ are both rational numbers.

\section{REFERENCES}

[1] I. N. BAKER AND F. GRoss, Further results on factorization of entire functions, Proc. Symp. Pure Math. Vol. 11 (Amer. Math. Soc. Providence R.I. 1968), 30-35.

[2] F. Gross, On factorization of meromorphic functions, Trans. Amer. Math. Soc. 131 (1968), 215-222.

[3] W. K. HAyman, Meromorphic functions, (Clarendon Press, Oxford, 1964).

$4]$ R. Nevanlinna, Le théorème de Picard-Borel et la théorie des fonctions méromorphes, (Gauthier Villars, Paris, 1929).

5 K. Nino ANd M. Ozawa, Deficiencies of an entire algebrold function, Kōdar Math. Sem. Rep. 22 (1970), 98-113.

6. M. OzAWA, On the zero-one set of an entire function, Ibid. 28 (1977), 311316.

[7] H. URABE, Uniqueness of the factorization under composition of certain entire functions, J. Math. Kyoto Univ. 18 (1978), 95-120.

8] H. URABE, A note on a certain functional equation concerning entire functions, Bull. Kyoto Univ. of Education Ser. B No. 51 (1977), 1-4.

[] H. URABE AND C.-C. YANG, On the zeros of an entire function which is periodic mod a non-constant entire function of order less than one, Proc. Japan Acad. Ser. A, 54 (1978), 142-144.

Departanent of Mathematics
Kyoto University of Educatiov,
Fusmim-he, Kyoto, 612, Japan.

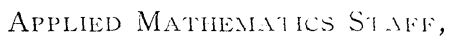
Naval Resfarcil Laboritory, Wasmivgton D. C. 20375.

Remark (added in the proof). About $*_{1}^{*}$ : Under $p_{\jmath}=p+c$, if in addition $p-q=$ const. $=c^{\prime}$, then using two relations $(※)$ in p. 261 , we represent $K(z)$ and $K\left(z+j b_{1}\right)$ as

$$
K(z)=L_{1}+L_{2} \cdot e^{-p}, \quad K\left(z+j b_{1}\right)=L_{3}+L_{4} \cdot e^{-p},
$$

where meromorphic functions $L_{\imath}(1 \leqq \imath \leqq 4)$ are all of order less than one. Since $K(z)$ is periodic with period $b_{2}$, by Lemma $\mathrm{B}$, we know that $L_{\imath}$ must be constant such that $L_{1}=L_{3}$ and $L_{2}=L_{4}$ (cf. around (30)). In view of these facts, by applying Lemma $\mathrm{B}$, we can conclude that $\left(R(z)\right.$ is constant or) $e^{c}=1$ and $e^{c^{\prime}}=1$. For instance, from $L_{1}=L_{3}$, if $R S_{j}-R_{j} S \neq \equiv$ (otherwise $R$ is constant), we deduce $\left(e^{c} S_{j}-S\right)\left(e^{c} k_{j} R_{j}-k R\right)=\left(e^{c} R_{j}-R\right)\left(e^{c} s_{j} S_{j}-s S\right) \quad$ so that $\quad\left(e^{c} k_{j} R_{j}-k R\right) /\left(e^{c} R_{j}-R\right)$ $=$ const. $=c^{\prime \prime}$ (say), which is rewritten as $e^{c}\left(k_{j}-c^{\prime \prime}\right) R_{j}=\left(k-c^{\prime \prime}\right) R$ (cf. the notations (10), (12) and (14)). Hence $\left(k-c^{\prime \prime}\right) R$ is constant $(\neq 0)$ so that we have $e^{c}=1$. Similarly, from $L_{2}=L_{4}$ we can conclude $e^{c^{\prime}}=1$. Thus $e^{p(z)}$ becomes doubly periodic, a contradiction.

About $*_{2}$ : More simply as above, we can show that $p-q \not$ const. In fact, if otherwise, using (48), we get also $e^{c}=e^{c^{\prime}}=1$ by Lemma B. 